\title{
Supporting Information for \\ Oxygen Is the High-Energy Molecule Powering Complex Multicellular Life: Fundamental Corrections to Traditional Bioenergetics
}

\author{
Klaus Schmidt-Rohr* \\ Department of Chemistry, Brandeis University, Waltham MA 02465, USA
}

ACS Omega, 2020

DOI: $10.1021 /$ acsomega. $9 \mathrm{~b} 03352$

*: srohr@brandeis.edu, (781) 736-2520

KEYWORDS: Biochemical energetics, chemical energy, aerobic respiration, electron transfer to oxygen, electron-transfer chain 


\section{Bond-energy analysis of combustion without assigning a bond order to $\mathbf{O}_{2}$. For readers} uncomfortable with assigning a bond order of two to $\mathrm{O}_{2}$, a slightly more complex description of the energetics of combustion or aerobic respiration than in the main text can be given: $\mathrm{O}_{2}$ is a high-energy molecule because its bond (whatever its bond order) is $306 \mathrm{~kJ} / \mathrm{mol}$ less stable than a $\mathrm{C}=\mathrm{O}$ bond (whatever its order) in the combustion product $\mathrm{CO}_{2}$. The bonds of oxygen formed with hydrogen, resulting in $\mathrm{H}_{2} \mathrm{O}$, are accounted for elsewhere, since based on hydrogen balance every pair of C-H or N-H bonds in the fuel is matched by the bonds in an $\mathrm{H}_{2} \mathrm{O}$ molecule, with a release of $107 \mathrm{~kJ}$. Similarly, a pair of C-C or C-O bonds converting to $\mathrm{C}=\mathrm{O}$ in $\mathrm{CO}_{2}$ releases $\sim 115 \mathrm{~kJ}$. The contributions from the bonds of the fuel thus average to $\sim 112 \mathrm{~kJ}$ per mole of $\mathrm{O}_{2}$, and a total heat of combustion of $-306-112=-418 \pm 10 \mathrm{~kJ}$ per mole of $\mathrm{O}_{2}$ results. The underlying rigorous mathematical analysis is presented in the next section.

This description leaves open the possibility that combustion could be exothermic because $\mathrm{CO}_{2}$ is particularly stable. This is not the case: The total bond energy of $\mathrm{CO}_{2}$ is equal (to within $<0.5 \%$ ) to that of $\mathrm{CH}_{4}$. The electron-pair bonds in $\mathrm{CO}_{2}$ are not unusual, being as strong as $\mathrm{C}-\mathrm{H}$, weaker than $\mathrm{O}-\mathrm{H}$, and stronger than $\mathrm{C}-\mathrm{O}$ bonds.

While this description is quantitative and accurate, it does not compare the energy of $\mathrm{O}_{2}$ and $\mathrm{H}_{2} \mathrm{O}$ directly. This can be remedied easily. The bond energies of $\mathrm{O}_{2}+2 \mathrm{H}_{2}$ and $2 \mathrm{H}_{2} \mathrm{O}(\mathrm{g})$ differ by $485 \mathrm{~kJ} / \mathrm{mol}$. Since the bonds in $2 \mathrm{H}_{2}$ are weaker than those in $\mathrm{H}_{2} \mathrm{O}$ by $2 \times(-28) \mathrm{kJ} / \mathrm{mol}, \mathrm{O}_{2}$ is more energetic than $\mathrm{H}_{2} \mathrm{O}(\mathrm{g})$ by $429 \mathrm{~kJ} / \mathrm{mol}$, consistent with Figure 1 of ref. ${ }^{1}$ and the analogous free-energy figure here (Figure 1a in the main text).

All these results can be summarized in a simple diagram like Figure 1a if a bond order of two is assigned to $\mathrm{O}_{2}$. Most chemists will not find this objectionable, since the bonding in $\mathrm{O}_{2}$ is shown as $\mathrm{O}=\mathrm{O}$ in most chemistry textbooks.

\section{Rigorous bond-energy analysis of combustion without assigning a bond order to $\mathrm{O}_{2}$. As a} basis for the semiquantitative analysis in the previous section, the following repeats the derivation of the heat of combustion using a generalized bond-energy analysis ${ }^{1}$ but without specifying the bond order of $\mathrm{O}_{2}$. Combustion of organic compounds containing $\mathrm{C}, \mathrm{H}$, and $\mathrm{O}$ can be written as

$$
\mathrm{C}_{c} \mathrm{H}_{h} \mathrm{O}_{o}+v_{\mathrm{O}_{2}} \mathrm{O}_{2} \rightarrow v_{\mathrm{CO}_{2}} \mathrm{CO}_{2}+v_{\mathrm{H}_{2} \mathrm{O}} \mathrm{H}_{2} \mathrm{O}
$$

The heat of combustion $\Delta_{c} H^{\circ}$ can be expressed in terms of bond-formation (negative bonddissociation) enthalpies ${ }^{1}$ :

$$
\begin{gathered}
\Delta_{\mathrm{c}} H^{\mathrm{o}} \approx v_{\mathrm{H}_{2} \mathrm{O}}(-927 \mathrm{~kJ} / \mathrm{mol})+v_{\mathrm{CO}_{2}} 2(-804 \mathrm{~kJ} / \mathrm{mol}) \\
-n_{\mathrm{CH}}(-410 \mathrm{~kJ} / \mathrm{mol})-n_{\mathrm{OH}}(-464 \mathrm{~kJ} / \mathrm{mol}) \\
-n_{\mathrm{no} \mathrm{H}} \bar{H}_{\mathrm{no} \mathrm{H}}-v_{\mathrm{O}_{2}}(-498 \mathrm{~kJ} / \mathrm{mol})
\end{gathered}
$$

where $n_{\mathrm{CH}}$ and $n_{\mathrm{OH}}$ are the numbers of $\mathrm{C}-\mathrm{H}$ and $\mathrm{O}-\mathrm{H}$ bonds, respectively, while $n_{\text {no } \mathrm{H}}$ is the number of the bonds in the fuel that do not involve hydrogen. $\bar{H}_{\text {nо H }} \approx-344 \pm 15 / \sqrt{ } n_{\text {no H }} \mathrm{kJ} / \mathrm{mol}$ (near the 
value of $-350 \mathrm{~kJ} / \mathrm{mol}$ for $\mathrm{C}-\mathrm{C}$ or $\mathrm{C}-\mathrm{O}$ bonds, ${ }^{2,3}$ which are the most common) is taken as an average value for the molar enthalpy of electron-pair bonds in the fuel, excluding $\mathrm{C}-\mathrm{H}$ and $\mathrm{O}-\mathrm{H}$ bonds. Hydrogen balance requires

$$
n_{\mathrm{CH}}+n_{\mathrm{OH}}=2 v_{\mathrm{H}_{2}} \mathrm{O}
$$

which can be rewritten as

$$
n_{\mathrm{CH}} / 2=v_{\mathrm{H}_{2} \mathrm{O}}-n_{\mathrm{OH}} / 2
$$

The unchanged number of electron-pair bonds before and after the reaction, assuming a bond order of two for $\mathrm{O}_{2}$, gave us ${ }^{1}$

$$
n_{\mathrm{CH}}+n_{\mathrm{OH}}+n_{\mathrm{no} \mathrm{H}}+2 v_{\mathrm{O}_{2}}=2 v_{\mathrm{H}_{2} \mathrm{O}}+4 v_{\mathrm{CO}_{2}}
$$

If we assumed that $\mathrm{O}_{2}$ has only one bond but gives rise to an additional bond in the products, the equivalent relation, with the 'extra-bond correction' $-v_{\mathrm{O} 2}$ on the side of the products,

$$
n_{\mathrm{CH}}+n_{\mathrm{OH}}+n_{\mathrm{noH}}+v_{\mathrm{O}_{2}}=2 v_{\mathrm{H}_{2} \mathrm{O}}+4 v_{\mathrm{CO}_{2}}-v_{\mathrm{O}_{2}}
$$

would be obtained. Generally, relation (S4) between integers characteristic of the molecular structures remains valid independent of the bond order assigned to $\mathrm{O}_{2}$.

According to eq.(S2), $n_{\mathrm{CH}}+n_{\mathrm{OH}}$ and $2 v_{\mathrm{H}_{2} \mathrm{O}}$ in eq.(S4) cancel and we retain

$$
2 v_{\mathrm{CO}_{2}}=n_{\mathrm{no} \mathrm{H}} / 2+v_{\mathrm{O}_{2}}
$$

After regrouping terms in eq.(S1) and using eq.(S6) one obtains:

$$
\begin{aligned}
\Delta_{\mathrm{c}} H^{\mathrm{o}} \mathrm{mol} & \approx\left(v_{\mathrm{H}_{2} \mathrm{O}}-n_{\mathrm{OH}} / 2\right)(-927 \mathrm{~kJ})+\left(n_{\mathrm{no} \mathrm{H}} / 2+v_{\mathrm{O}_{2}}\right)(-804 \mathrm{~kJ}) \\
& -n_{\mathrm{CH}}(-410 \mathrm{~kJ})-n_{\mathrm{no} \mathrm{H}}\left(-344 \pm 15 / \sqrt{ } n_{\mathrm{noH}}\right) \mathrm{kJ}-v_{\mathrm{O}_{2}}(-498 \mathrm{~kJ})
\end{aligned}
$$

which can be further simplified by combining terms and using eq.(S3) for the first factor:

$$
\begin{aligned}
\Delta_{\mathrm{c}} H^{\mathrm{o}} \mathrm{mol} \approx & n_{\mathrm{CH}} / 2(-927 \mathrm{~kJ})+n_{\mathrm{no} \mathrm{H}}\left(-344 \pm 15 / \sqrt{ } n_{\mathrm{no} \mathrm{H}}-804 / 2\right) \mathrm{kJ} \\
& -n_{\mathrm{CH}}(-410 \mathrm{~kJ})+v_{\mathrm{O}_{2}}(-804 \mathrm{~kJ}+498 \mathrm{~kJ}) \\
= & n_{\mathrm{CH}}(-53 \mathrm{~kJ})+n_{\mathrm{no} \mathrm{H}}\left(-58 \pm 15 / \sqrt{ } n_{\mathrm{noH}}\right) \mathrm{kJ}+\mathrm{v}_{\mathrm{O}_{2}}(-306 \mathrm{~kJ})
\end{aligned}
$$

The main term here is the stoichiometric coefficient of $\mathrm{O}_{2}$ multiplied by the difference $-804 \mathrm{~kJ}+$ $498 \mathrm{~kJ}=-306 \mathrm{~kJ}$ between the $\mathrm{O}_{2}$ and carbon dioxide $\mathrm{C}=\mathrm{O}$ bond energies, without any assumption about the bond orders.

Disregarding the relatively small difference between -53 and $-58 \mathrm{~kJ} / \mathrm{mol}$ in eq.(S7), setting 
both equal to $-56 \mathrm{~kJ} / \mathrm{mol}$, eq.(S7) simplifies to

$$
\Delta_{\mathrm{c}} H^{\mathrm{o}} \approx\left(n_{\mathrm{CH}}+n_{\mathrm{no} \mathrm{H}}\right)(-56 \mathrm{~kJ} / \mathrm{mol})+v_{\mathrm{O}_{2}}(-306 \mathrm{~kJ} / \mathrm{mol})
$$

The coefficient $\left(n_{\mathrm{CH}}+n_{\text {no }}\right)$ is nearly proportional to $v_{\mathrm{O}_{2}}$, since the number of bonds in the fuel and the amount of $\mathrm{O}_{2}$ used in the combustion increase nearly proportionally. From oxygen balance and eqs.(S4) and (S2), we find

$$
\begin{aligned}
2 v_{\mathrm{O}_{2}}+o=v_{\mathrm{H}_{2} \mathrm{O}}+2 v_{\mathrm{CO}_{2}}= & n_{\text {bonds }} / 2=n_{\mathrm{H}} / 2+n_{\mathrm{noH}} / 2+v_{\mathrm{O}_{2}} \\
& =n_{\mathrm{CH}} / 2+n_{\mathrm{OH}} / 2+n_{\mathrm{noH}} / 2+v_{\mathrm{O}_{2}}
\end{aligned}
$$

so

$$
n_{\mathrm{CH}}+n_{\mathrm{no}}=2 v_{\mathrm{O}_{2}}+2 o-n_{\mathrm{OH}} \approx 2 v_{\mathrm{O}_{2}}
$$

which holds exactly for hydrocarbons, where $o=0$ and $n_{\mathrm{OH}}=0$, while for carbohydrates, $+2 o$ and $n_{\text {Он }}$ partially offset each other. Also because of the relatively small magnitude, $56 \mathrm{~kJ} / \mathrm{mol}$, of the first term in eq.(S8), we can generally approximate $\left(n_{\mathrm{CH}}+n_{\mathrm{noH}}\right)$ by $2 v_{\mathrm{O}_{2}}$ in eq.(S8) to obtain

$$
\Delta_{\mathrm{c}} H^{\circ} \approx 2 v_{\mathrm{O}_{2}}(-56 \mathrm{~kJ} / \mathrm{mol})+v_{\mathrm{O}_{2}}(-306 \mathrm{~kJ} / \mathrm{mol})=(-418 \mathrm{~kJ} / \mathrm{mol}) v_{\mathrm{O}_{2}}
$$

which is in excellent agreement with the experimental data for hundreds of organic molecules. ${ }^{1}$

The weak $\boldsymbol{\sigma}$-bond in $\mathbf{O}_{2}$. The relative weakness of the double bond in $\mathrm{O}_{2}$, which makes it a highenergy molecule, can be attributed to its weak $\sigma$-bond. ${ }^{4}$ Single O-O, O-F, F-F, O-N, and N-N bonds (e.g. in $\mathrm{H}_{2} \mathrm{O}_{2}$, peroxides, and $\mathrm{F}_{2}$ ) have dissociation energies of only $150-220 \mathrm{~kJ} / \mathrm{mol},{ }^{2,3,5}$ less than half of the bond energy of $\mathrm{O}_{2}(498 \mathrm{~kJ} / \mathrm{mol})$; these bonds are also clearly weaker than the $\mathrm{O}-\mathrm{H}, \mathrm{H}-\mathrm{H}, \mathrm{C}-\mathrm{H}, \mathrm{N}-\mathrm{H}, \mathrm{C}-\mathrm{C}$, and C-O single bonds $(460-350 \mathrm{~kJ} / \mathrm{mol})$ in organic fuel molecules and $\mathrm{H}_{2}$. Borden et al. have tentatively deduced a dissociation energy of only $80 \mathrm{~kJ} / \mathrm{mol}$ (see footnote 57 in ref. ${ }^{4}$ ) for the $\sigma$-bond in $\mathrm{O}_{2}$. While the triplet $\pi$-electron system of $\mathrm{O}_{2}$ is unusual and complicated, it contributes more strongly to the bonding in $\mathrm{O}_{2}$ than does the $\sigma$-bond. ${ }^{4}$ According to Pauling, the $\pi$-electrons give rise to two three-electron bonds, whose bond energy is generally "about one-half that of a single bond". ${ }^{6}$ With two "half $\pi$-bonds' and a single $\sigma$-bond, representing $\mathrm{O}_{2}$ with a double bond seems as justified as any simple alternative. A double bond also simplifies a generalized bond-energy analysis, which is easiest if the number of bonds does not change during the reaction. ${ }^{1}$ Borden et al. have deduced that the bonding by the two three-electron $\pi$ bonds is further stabilized by resonance between them, which contributes to the kinetic stability (persistence) of $\mathrm{O}_{2}$ in the atmosphere. ${ }^{4}$ 
Table S1. Enthalpies and Gibbs free energies of simple molecules and ions relevant in biochemical energetics, in $\mathrm{kJ} / \mathrm{mol}$. Left two columns: traditional enthalpies and Gibbs energies of formation, which are not energies of just the species named but also depend on the bond energies of the elements in their standard states, and in the case of ions on the hydration energy of $z$ protons, where $z$ is the ion charge number. Right two columns: Natural individual enthalpies and Gibbs energies, dominated by bond energies of the molecule named, and/or ionization energy in water in the case of an ion.

\section{Thermodynamic Energies for Biochemistry: Conventional vs. Individual}

Traditional

(' $\mathrm{H}^{+}(\mathrm{aq})$ defined as $0 \mathrm{~kJ} / \mathrm{mol}$ ')

\begin{tabular}{lcc}
\multicolumn{1}{c}{$\Delta_{\mathrm{f}} H^{\mathrm{o}}(\mathrm{kJ} / \mathrm{mol})$} & $\Delta_{\mathrm{f}} G^{\mathrm{o}}(\mathrm{kJ} / \mathrm{mol})$ \\
$\mathrm{H}_{2}(\mathrm{~g})$ & 0 & 0 \\
$\mathrm{~F}_{2}(\mathrm{~g})$ & 0 & 0 \\
$\mathrm{Cl}_{2}(\mathrm{~g})$ & 0 & 0 \\
$\mathrm{O}_{2}(\mathrm{~g})$ & 0 & 0 \\
$\mathrm{~N}_{2}(\mathrm{~g})$ & 0 & 0 \\
$\mathrm{CO}_{2}(\mathrm{~g})$ & -394 & -394 \\
$\mathrm{CH}_{4}(\mathrm{~g})$ & -75 & -51 \\
$\mathrm{HCl}(\mathrm{g})$ & -92 & -95 \\
$\mathrm{H}_{2} \mathrm{O}(l)$ & -286 & -237 \\
$\mathrm{MetOH}(l)$ & -239 & -166 \\
$\mathrm{MetOH}(\mathrm{aq})$ & -245 & -175 \\
EtOH$(l)$ & -278 & -175 \\
EtOH(aq) & -288 & -182 \\
$\mathrm{CH} \mathrm{CO}_{2} \mathrm{H}(\mathrm{aq})-486$ & -396 \\
Glucose(s) & -1275 & -911 \\
Glucose(aq) & -1264 & -917
\end{tabular}

Meaningful individual enthalpy \& free-energy values (relative to the free atoms)

$\begin{array}{lll} & H^{\mathrm{o}} .(\mathrm{kJ} / \mathrm{mol}) & G^{\mathrm{o}} \ldots(\mathrm{kJ} / \mathrm{mol}) \\ \mathrm{H}_{2}(\mathrm{~g}) & -436 \text { (bond) } & \mathbf{- 4 0 6 . 5} \\ \mathrm{F}_{2}(\mathrm{~g}) & -158 \text { (bond) } & \mathbf{- 1 2 3 . 9} \\ \mathrm{Cl}_{2}(\mathrm{~g}) & -242 \text { (bond) } & \mathbf{- 2 1 1 . 4} \\ \mathrm{O}_{2}(\mathrm{~g}) & -498 \text { (bond) } & \mathbf{- 4 6 3 . 5} \\ \mathrm{N}_{2}(\mathrm{~g}) & -945.4 \text { (bond) } & \mathbf{- 9 1 1 . 2} \\ \mathrm{CO}_{2}(\mathrm{~g}) & -1608 \text { (bonds) } & \mathbf{- 1 5 2 9} \\ \mathrm{CH}_{4}(\mathrm{~g}) & -1630 \text { (bonds) } & \mathbf{- 1 5 3 5} \\ \mathrm{HCl}(\mathrm{g}) & -431 \text { (bond) } & \mathbf{- 4 0 4 . 3} \\ \mathrm{H}_{2} \mathrm{O}(l) & -971 \text { (bonds+cond.) } & \mathbf{- 8 7 5 . 5} \\ \mathrm{tOH}(l) & -2077 \text { (bonds+cond.) } & \mathbf{- 1 8 8 1 . 8} \\ \mathrm{tOH}(\mathrm{aq}) & -2083 \text { (bonds+hydr.) } & \mathbf{- 1 8 9 0 . 8} \\ \mathrm{H}(l) & -3269 \text { (bonds }+ \text { cond.) } & \mathbf{- 2 9 6 8} \\ \mathrm{H}(\mathrm{aq}) & -3279 \text { (bonds }+ \text { hydr.) } & \mathbf{- 2 9 7 5} \\ \mathrm{O}_{2} \mathrm{H}(\mathrm{aq}) & -3290 \text { ( bonds) } & \mathbf{- 3 0 1 4 . 5} \\ \mathrm{cose}(\mathrm{s}) & -9672 \text { ( bonds) } & \mathbf{- 8 7 6 7} \\ \mathrm{cose}(\mathrm{aq}) & -9661 \text { (bonds+hydr.) } & \mathbf{- 8 7 7 4}\end{array}$

\begin{tabular}{|c|c|c|c|c|c|}
\hline $\mathrm{H}^{+}(\mathrm{aq})$ & 0 & 0 & $\mathrm{H}^{+}(\mathrm{aq})$ & +162 (IE+hydr.) & 210 \\
\hline $\mathrm{H}^{+}(\mathrm{pH} 7)$ & 0 & $R T \ln 10^{-7}=-40$ & $\mathrm{H}^{+}(\mathrm{pH} 7)$ & +162 (IE+hydr.) & 170 \\
\hline $\mathrm{Li}^{+}(\mathrm{aq})$ & -278.5 & -293 & $\mathrm{Li}^{+}(\mathrm{aq})$ & -58 (IE+hydr.) & -7 \\
\hline $\mathrm{Na}^{+}(\mathrm{aq})$ & -240.3 & -262 & $\mathrm{Na}^{+}(\mathrm{aq})$ & +33 (IE+hydr.) & 74 \\
\hline $\mathrm{K}^{+}(\mathrm{aq})$ & -252.1 & -283.3 & $\mathrm{~K}^{+}(\mathrm{aq})$ & +38 (IE+hydr.) & 69 \\
\hline $\mathrm{Ag}^{+}(\mathrm{aq})$ & 105.8 & 77 & $\mathrm{Ag}^{+}(\mathrm{aq})$ & +201 (IE+hydr.) & 244 \\
\hline $\mathrm{Mg}^{2+}(\mathrm{aq})$ & -467 & -455 & $\mathrm{Mg}^{2+}(\mathrm{aq})$ & +146 (IE+hydr.) & 257 \\
\hline $\mathrm{Ca}^{2+}(\mathrm{aq})$ & -543 & -554 & $\mathrm{Ca}^{2+}(\mathrm{aq})$ & +39 (IE+hydr.) & 127 \\
\hline $\mathrm{Fe}^{2+}(\mathrm{aq})$ & -89.1 & -78.9 & $\mathrm{Fe}^{2+}(\mathrm{aq})$ & +254 (IE+hydr.) & 368 \\
\hline $\mathrm{Co}^{2+}(\mathrm{aq})$ & -58 & -54.4 & $\mathrm{Co}^{2+}(\mathrm{aq})$ & +277 (IE+hydr.) & 391 \\
\hline $\mathrm{Ni}^{2+}(\mathrm{aq})$ & -54 & -45.6 & $\mathrm{Ni}^{2+}(\mathrm{aq})$ & +276 (IE+hydr.) & 395 \\
\hline $\mathrm{Cu}^{2+}(\mathrm{aq})$ & +64.8 & 65.5 & $\mathrm{Cu}^{2+}(\mathrm{aq})$ & +486 (IE+hydr.) & 592 \\
\hline $\mathrm{Zn}^{2+}(\mathrm{aq})$ & -153.4 & -147 & $\mathrm{Zn}^{2+}(\mathrm{aq})$ & +475 (IE+hydr.) & 583 \\
\hline $\mathrm{Cd}^{2+}(\mathrm{aq})$ & -75.9 & & $\mathrm{Cd}^{2+}(\mathrm{aq})$ & +572 (IE+hydr.) & 670 \\
\hline $\mathrm{Hg}^{2+}(\mathrm{aq})$ & +171.1 & & $\mathrm{Hg}^{2+}(\mathrm{aq})$ & +870 (IE+hydr.) & 958 \\
\hline $\mathrm{Pb}^{2+}(\mathrm{aq})$ & -1.7 & -24.4 & $\mathrm{~Pb}^{2+}(\mathrm{aq})$ & +563 (IE +hydr.) & 639 \\
\hline $\mathrm{Al}^{3+}(\mathrm{aq})$ & -538.4 & -485 & $\mathrm{Al}^{3+}(\mathrm{aq})$ & +283 (IE+hydr.) & 467 \\
\hline $\mathrm{Fe}^{3+}(\mathrm{aq})$ & -48.5 & -4.7 & $\mathrm{Fe}^{3+}(\mathrm{aq})$ & (IE+hydr.) & 856 \\
\hline $\mathrm{F}^{-}(\mathrm{aq})$ & -333 & -279 & $\mathrm{~F}^{-}(\mathrm{aq})$ & -792 (-EA+hydr.) & -752 \\
\hline $\mathrm{Cl}^{-}(\mathrm{aq})$ & -167.1 & -131 & $\mathrm{Cl}^{-}(\mathrm{aq})$ & -669 (-EA+hydr.) & -650 \\
\hline $\mathrm{OH}^{-}(\mathrm{aq})$ & -230 & -157 & $\mathrm{OH}^{-}(\mathrm{aq})$ & -1076 (bond- $E A+$ hydr. & ) -1006 \\
\hline $\mathrm{C}$ (graphi & ite) 0 & 0 & $\mathrm{C}$ (graphi & e) -717 ( 2 bonds/C) & -671 \\
\hline
\end{tabular}




$\begin{array}{lllllll}\mathrm{Fe}(\mathrm{s}) & 0 & 0 & \mathrm{Fe}(\mathrm{s}) & -416 \text { (cohesive) } & -370.7 & I E: 2320 \\ \mathrm{Cu}(\mathrm{s}) & 0 & 0 & \mathrm{Cu}(\mathrm{s}) & -338 \text { (cohesive) } & -298.6 & I E: 2703 \\ \mathrm{Zn}(\mathrm{s}) & 0 & 0 & \mathrm{Zn}(\mathrm{s}) & -131 \text { (cohesive) } & -95.2 & \text { IE: } 2639\end{array}$

"bond" = bond energy; "cond." = $-\Delta_{\text {vap }} H^{\circ}$

"IE" = (total) ionization energy; "hydr." = hydration/solvation energy; " $E A "=$ electron affinity

"IE+hydr." = ionization energy in water

"cohesive" = lattice cohesive energy = crystal bonding energy = energy released when the atoms come together to form the crystal $=-\Delta_{\text {subl }} H^{0}=-\Delta_{\mathrm{f}} H^{\mathrm{o}}$ of the gaseous atom.

Note that in the $\Delta_{\mathrm{r}} G^{0}$ "values given in the text, typically only three figures are significant.

Origin of data for Table S1 and Figure 1. Some data were given in ref., while others were calculated as follows, from widely tabulated free energies of formation ${ }^{8}$ based on the formation reaction:

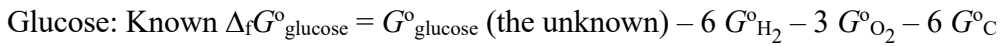

In $\mathrm{kJ} / \mathrm{mol}:-911=G_{\text {glucose }}^{\mathrm{o}}-6(-406.5)-3(-463.5)-6(-671)$

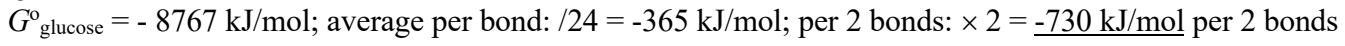

$\mathrm{CO}_{2}$ : Known $\Delta_{\mathrm{f}} G^{\mathrm{o}} \mathrm{CO} 2=G^{\mathrm{o}} \mathrm{CO}_{2}$ (the unknown) $-G_{\mathrm{O}_{2}}-G_{\mathrm{C}}$

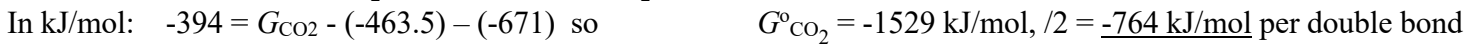

$\mathrm{CH}_{4}$ : Known $\Delta_{\mathrm{f}} G^{\mathrm{o}} \mathrm{CH}_{4}=G^{\mathrm{o}} \mathrm{CH}_{4}$ (the unknown) $-2 G^{\mathrm{o}}{ }_{\mathrm{H}_{2}}-G_{\mathrm{C}^{\mathrm{o}}}$

In kJ/mol: $\quad-51=G_{\mathrm{CH}_{4}}^{\mathrm{o}}-2(-406.5)-(-671)$ so $\quad G_{\mathrm{CH}_{4}}^{\mathrm{o}}=-1535 \mathrm{~kJ} / \mathrm{mol}$; per 2 bonds: $/ 2=\underline{-768 \mathrm{~kJ} / \mathrm{mol}}$ per $2 \mathrm{bonds}$

Methanol(aq): Known $\Delta_{\mathrm{f}} G^{\mathrm{o}}{ }_{\mathrm{MetOH}(\mathrm{aq})}=G^{\mathrm{o}}{ }_{\mathrm{MetOH}}\left(\right.$ the unknown) $-2 G_{\mathrm{H}_{2}}^{\mathrm{o}}-1 / 2 G_{\mathrm{O}_{2}}^{\mathrm{o}}-G^{\mathrm{o}} \mathrm{C}$

In kJ/mol: $-175=G^{\mathrm{o}}{ }_{\mathrm{MetOH}}$ (the unknown) $-2(-406.5)-1 / 2(-463.5)-(-671)$

$G^{\mathrm{o}}{ }_{\mathrm{MetOH}}=-1891 \mathrm{~kJ} / \mathrm{mol}$, average per bond: $/ 5=-378 \mathrm{~kJ} / \mathrm{mol}$; per 2 bonds: $\times 2=\underline{-756 \mathrm{~kJ} / \mathrm{mol}}$ per 2 bonds

Ethanol(aq): Known $\Delta_{\mathrm{f}} G^{\mathrm{o}}{ }_{\text {EtOH(aq) }}=G^{\mathrm{o}}{ }_{\mathrm{EtOH}}$ (the unknown) $-3 G_{\mathrm{H}_{2}}^{\mathrm{o}}-1 / 2 G^{\mathrm{o}} \mathrm{O}_{2}-2 G^{\mathrm{o}} \mathrm{C}$

In kJ/mol: $-182=G^{0}{ }_{\text {EtOH }}$ (the unknown) $-3(-406.5)-1 / 2(-463.5)-2(-671)$

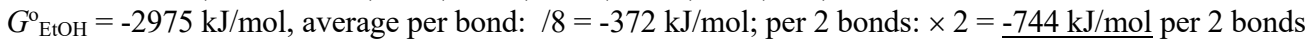

Acetic acid (aq): Known $\Delta_{\mathrm{f}} G^{\mathrm{o}} \mathrm{Ac}(\mathrm{aq})=G^{\mathrm{o}}$ Ac (the unknown) $-2 G_{\mathrm{H}_{2}}^{\mathrm{o}}-G^{\mathrm{o}} \mathrm{O}_{2}-2 G^{\mathrm{o}}{ }_{\mathrm{C}}$

In kJ/mol: $\quad-396=G^{\mathrm{o}}{ }_{\mathrm{Ac}}$ (the unknown) $-2(-436)-(-463.5)-2(-671)$

$G^{\mathrm{o}}{ }_{\mathrm{Ac}}=-3014.5 \mathrm{~kJ} / \mathrm{mol}$, average per electron-pair bond: $/ 8=-377 \mathrm{~kJ} / \mathrm{mol}$; per 2 bonds: $\times 2=\underline{-754 \mathrm{~kJ} / \mathrm{mol}}$ per 2 bonds

Glyceraldehyde 3-phosphate: $\mathrm{C}_{3} \mathrm{H}_{7} \mathrm{O}_{6} \mathrm{P}$

Known $\quad \Delta_{\mathrm{f}} G^{\mathrm{o} "}{ }_{\mathrm{G} 3 \mathrm{P}}-3.5 \Delta_{\mathrm{f}} G^{\mathrm{o}{ }_{\mathrm{H}}}=G_{\mathrm{G} 3 \mathrm{P}}^{\mathrm{o}}\left(\right.$ the unknown) $-3.5 G_{\mathrm{H}_{2}}^{\mathrm{o}}-3 G_{\mathrm{O}_{2}}^{\mathrm{o}}-3 G^{\mathrm{o}}{ }_{\mathrm{C}}-G_{\mathrm{P}}^{\mathrm{o}}$

In $\mathrm{kJ} / \mathrm{mol}: \quad-1077-3.5 \Delta_{\mathrm{f}} G^{\mathrm{o}}{ }_{\mathrm{H} 2}=G_{\mathrm{G} 3 \mathrm{P}}^{\mathrm{o}}-3.5(-406.5)-3(-463.5)-3(-671)-(-278.3)$

$-1077-3.5 \times 82=-1364=G^{o}{ }_{\mathrm{G} 3 \mathrm{P}}+5105$

Pyruvic acid: $\mathrm{C}_{3} \mathrm{H}_{4} \mathrm{O}_{3}$

$G_{\mathrm{G} 3 \mathrm{P}}^{\mathrm{o}}=-6469 \mathrm{~kJ} / \mathrm{mol}$; average per bond: $/ 18=-359 \mathrm{~kJ} / \mathrm{mol}$; per 2 bonds: $\times 2=\underline{-718 \mathrm{~kJ} / \mathrm{mol}}$ per 2 bonds

Known $^{9} \quad \Delta_{\mathrm{f}} G_{\text {Pyr }}^{\mathrm{o}}=G^{\mathrm{o}}$ Pyr $($ the unknown $)-2 G_{\mathrm{H}_{2}}^{\mathrm{o}}-1.5 G^{\mathrm{o}} \mathrm{O}_{2}-3 G^{\mathrm{o}}$

In kJ/mol: $\quad-489=G^{\mathrm{o}} \mathrm{Pyr}-2(-406.5)-1.5(-463.5)-3(-671)$

$-489=G_{\text {Pyr }}^{\mathrm{o}}+3521$

$G_{\text {Pyr }}^{\mathrm{o}}=-4010 \mathrm{~kJ} / \mathrm{mol}$; average per bond: $/ 11=-365 \mathrm{~kJ} / \mathrm{mol}$; per 2 bonds: $\times 2=-729 \mathrm{~kJ} / \mathrm{mol}$ per 2 bonds

$\mathrm{H}_{3} \mathrm{PO}_{4}(\mathrm{aq}): \mathrm{Known}^{9} \Delta_{\mathrm{f}} G_{\mathrm{PA}}^{\mathrm{o}}=G_{\mathrm{PA}}^{\mathrm{o}}$ (the unknown) - $1.5 G_{\mathrm{H}_{2}}^{\mathrm{o}}-2 G_{\mathrm{O}_{2}}^{\mathrm{o}}-G_{\mathrm{P}}^{\mathrm{o}}$

In kJ/mol: $-1142=G_{\mathrm{PA}}^{\mathrm{o}}-1.5(-406.5)-2(-463.5)-(-278.3)=G_{\mathrm{PA}}^{\mathrm{o}}+1815$

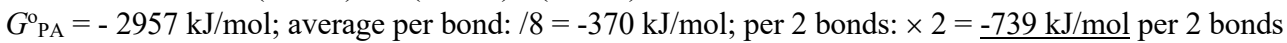

Enthalpies:

Glucose: Known $\Delta_{\mathrm{f}} H^{\mathrm{o}}$ glucose $=H^{\mathrm{o}}$ glucose (the unknown) $-6 H_{\mathrm{H}_{2}}^{\mathrm{o}}-3 H^{\mathrm{o}} \mathrm{O}_{2}-6 H^{\mathrm{o}} \mathrm{C}$

In $\mathrm{kJ} / \mathrm{mol}:-1275=H_{\text {glucose }}^{\mathrm{o}}-6(-436)-3(-498)-6(-717)$

$H_{\text {glucose }}^{\mathrm{o}}=-9687 \mathrm{~kJ} / \mathrm{mol}$; average per bond: $/ 24=-404 \mathrm{~kJ} / \mathrm{mol}$;

per 2 bonds: $\times 2=\underline{-807 \mathrm{~kJ} / \mathrm{mol}}$ per 2 bonds (many C-C \& C-O at $-700 \mathrm{~kJ} / \mathrm{mol}$ ) 


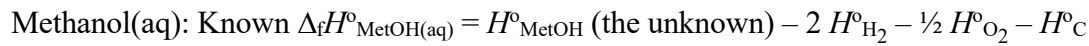

In $\mathrm{kJ} / \mathrm{mol}:-245=H^{\mathrm{o}} \mathrm{MetOH}$ (the unknown) $-2(-436)-1 / 2(-498)-(-717)$

$H^{\mathrm{o}}{ }_{\mathrm{MetOH}}=-2083 \mathrm{~kJ} / \mathrm{mol}$, average per bond: $/ 5=-417 \mathrm{~kJ} / \mathrm{mol}$; per 2 bonds: $\times 2=\underline{-833 \mathrm{~kJ} / \mathrm{mol}}$ per 2 bonds

Ethanol(aq): Known $\Delta_{\mathrm{f}} H_{\mathrm{EtOH}(\mathrm{aq})}^{\mathrm{o}}=H_{\mathrm{EtOH}}^{\mathrm{o}}$ (the unknown) $-3 H_{\mathrm{H}_{2}}^{\mathrm{o}}-1 / 2 H^{\mathrm{o}} \mathrm{O}_{2}-2 H^{\mathrm{o}} \mathrm{C}$

In $\mathrm{kJ} / \mathrm{mol}:-288=H^{\mathrm{o}}{ }_{\mathrm{EtOH}}$ (the unknown) $-3(-436)-1 / 2(-498)-2(-717)$

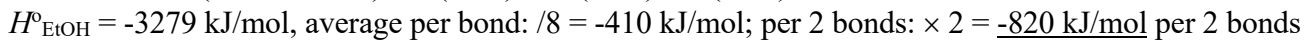

Acetic acid (aq): Known $\Delta_{\mathrm{f}} H^{\mathrm{o}} \mathrm{Ac}(\mathrm{aq})=H^{\mathrm{o}}$ Ac (the unknown) $-2 H^{\mathrm{o}} \mathrm{H}_{2}-H^{\mathrm{o}} \mathrm{O}_{2}-2 H^{\circ}{ }_{\mathrm{C}}$

In $\mathrm{kJ} / \mathrm{mol}:-486=H_{\mathrm{Ac}}^{\mathrm{o}}$ (the unknown) $-2(-436)-(-498)-2(-717)$

$H^{\mathrm{o}} \mathrm{Ac}=-3290 \mathrm{~kJ} / \mathrm{mol}$, average per electron-pair bond: $/ 8=-411 \mathrm{~kJ} / \mathrm{mol}$; per 2 bonds: $\times 2=\underline{-823 \mathrm{~kJ} / \mathrm{mol}}$ per 2 bonds

Glyceraldehyde: $\mathrm{C}_{3} \mathrm{H}_{6} \mathrm{O}_{3}=\left(\mathrm{CH}_{2} \mathrm{O}\right)_{3}$ : The free energy of formation had to be estimated from various data.

(i) Starting with enthalpies of formation from Domalski: ${ }^{10}$

Glucose $\left(\mathrm{C}_{6} \mathrm{H}_{12} \mathrm{O}_{6}\right)$ : $\quad \Delta_{\mathrm{f}} H^{\mathrm{o}}=-303 \mathrm{kcal} / \mathrm{mol}=-1267 \mathrm{~kJ} / \mathrm{mol}$

Glyceraldehyde $\left(\mathrm{C}_{3} \mathrm{H}_{6} \mathrm{O}_{3}\right): \quad \Delta_{\mathrm{f}} H^{0}=\mathbf{- 1 4 3} \mathrm{kcal} / \mathrm{mol}=\mathbf{- 5 9 8} \mathrm{kJ} / \mathrm{mol}$

Lactic acid $\left(\mathrm{C}_{3} \mathrm{H}_{6} \mathrm{O}_{3}\right): \quad \Delta_{\mathrm{f}} H^{\circ}=-166 \mathrm{kcal} / \mathrm{mol}=-694 \mathrm{~kJ} / \mathrm{mol}$

Glyceraldehyde has a $96 \mathrm{~kJ} / \mathrm{mol}$ higher energy than its isomer lactic acid due to the weaker $\mathrm{C}-\mathrm{H}$ bond of an aldehyde.

Glycerol $\left(\mathrm{C}_{3} \mathrm{H}_{8} \mathrm{O}_{3}\right): \quad \Delta_{\mathrm{f}} H^{\circ}=-160 \mathrm{kcal} / \mathrm{mol}=-669 \mathrm{~kJ} / \mathrm{mol}$

Pyruvic acid $\left(\mathrm{C}_{3} \mathrm{H}_{4} \mathrm{O}_{3}\right): \quad \Delta_{\mathrm{f}} H^{\mathrm{o}}=-140.3 \mathrm{kcal} / \mathrm{mol}=-586.5 \mathrm{~kJ} / \mathrm{mol}$

$\begin{array}{lrl}\text { Glc } \rightarrow \text { 2 glyceraldehyde: } & \Delta_{\mathrm{r}} H^{\mathrm{o}}=\mathbf{2}(-\mathbf{5 9 8} \mathbf{~ k J} / \mathbf{m o l})-(-\mathbf{1 2 6 7} \mathbf{~ k J} / \mathbf{m o l})=+71 \mathbf{~ k J} / \mathbf{m o l} \\ \mathrm{Glc} \rightarrow 2 \text { lactic acid: } & \Delta_{\mathrm{r}} H^{\mathrm{o}}=2(-694 \mathrm{~kJ} / \mathrm{mol})-(-1267 \mathrm{~kJ} / \mathrm{mol})=-121 \mathrm{~kJ} / \mathrm{mol} \\ \mathrm{Glc}+2 \mathrm{H} \mathrm{H}_{2} \rightarrow 2 \text { glycerol: } & \Delta_{\mathrm{r}} H^{\mathrm{o}}=2(-669 \mathrm{~kJ} / \mathrm{mol})-(-1267 \mathrm{~kJ} / \mathrm{mol})=-71 \mathrm{~kJ} / \mathrm{mol} \\ \mathrm{Glc} \rightarrow 2 \text { pyruvic acid }+2 \mathrm{H}_{2}: \Delta_{\mathrm{r}} H^{\mathrm{o}}=2(-586.5 \mathrm{~kJ} / \mathrm{mol})-(-1267 \mathrm{~kJ} / \mathrm{mol})=+94 \mathrm{~kJ} / \mathrm{mol}\end{array}$

Gibbs free energy of formation:

Glc $\rightarrow 2$ lactic acid:

$-9172(-540) \quad \Rightarrow-163 \mathrm{~kJ} / \mathrm{mol}=\Delta_{\mathrm{r}} G^{\mathrm{o}}\left(\right.$ ref. $\left.^{9}\right)$

$\mathrm{Glc}+2 \mathrm{H}_{2} \rightarrow 2$ glycerol

$-427+2 \times 81.52(-171) \Rightarrow-78 \mathrm{~kJ} / \mathrm{mol}=\Delta_{\mathrm{r}} G^{\mathrm{o}}$

$-917+0 \quad 2(-477) \Rightarrow-37 \mathrm{~kJ} / \mathrm{mol}=\Delta_{\mathrm{r}} G^{\mathrm{o}} \quad\left(\right.$ ref. $\left.^{9}\right)$

Glc $\rightarrow 2$ pyruvic acid $+2 \mathrm{H}_{2}$ :

$-427 \quad 2(-351)+2 \times 81.5 \quad-112 \mathrm{~kJ} / \mathrm{mol}=\Delta_{\mathrm{r}} G^{\mathrm{o}}$

$-9172(-489)+0 \quad \Rightarrow \quad-61 \mathrm{~kJ} / \mathrm{mol}=\Delta_{\mathrm{r}} G^{\mathrm{o}} \quad\left(\right.$ ref. $\left.^{9}\right)$

Assuming similar entropy of both isomers of $\mathrm{C}_{3} \mathrm{H}_{6} \mathrm{O}_{3}$ :

Estimating $\Delta_{\mathrm{f}} G^{\mathrm{o}}$ glyceraldehyde

$$
\begin{aligned}
& \Delta_{\mathrm{f}} G_{\text {glyceraldehyde }}^{\mathrm{o}}-\Delta_{\mathrm{f}} G_{\text {lactic acid }}^{\mathrm{o}} \approx \Delta_{\mathrm{f}} H_{\text {glyceraldehyde }}^{\mathrm{o}}-\Delta_{\mathrm{f}} H^{\mathrm{o}} \text { lactic acid }=96 \mathrm{~kJ} / \mathrm{mol} \\
& \Delta_{\mathrm{f}} G_{\text {glyceraldehyde }}^{\mathrm{o}} \approx-540 \mathrm{~kJ} / \mathrm{mol}+96 \mathrm{~kJ} / \mathrm{mol}=-444 \mathrm{~kJ} / \mathrm{mol}
\end{aligned}
$$

(iia) Glycerol - glycerol 3-phosphate: $\Delta G^{\mathrm{o}}=-171+1077=906 \mathrm{~kJ} / \mathrm{mol}$

Glyceraldehyde - glyceraldehyde 3-phosphate: $\Delta G^{\mathrm{o}}=906 \mathrm{~kJ} / \mathrm{mol}$ assumed based on above

$\Delta_{\mathrm{f}} G^{\mathrm{o}}{ }_{\text {glyceraldehyde }}+1088 \mathrm{~kJ} / \mathrm{mol}=906 \mathrm{~kJ} / \mathrm{mol}$

$\Delta_{\mathrm{f}} G^{0}$ " ${ }_{\text {glyceraldehyde }}=-182 \mathrm{~kJ} / \mathrm{mol}$

(iib) 2 Glyceraldehyde 3-phosphate $\rightarrow$ Fructose 1,6-phosphate
$2(-1077)=-2154$
$-2207$
$\Delta_{\mathrm{r}} G^{\mathrm{o}}=-53 \mathrm{~kJ} / \mathrm{mol}$ : Higher energy of G3P

Assume same $\Delta_{\mathrm{r}} G^{\mathrm{o}}=-53 \mathrm{~kJ} / \mathrm{mol}$ without phosphates:

2 Glyceraldehyde $\rightarrow$ Fructose (or glucose)

$2 \Delta_{\mathrm{f}} G^{\mathrm{o}}$ "glyceraldehyde $\quad-426 \mathrm{~kJ} / \mathrm{mol}$

$-426 \mathrm{~kJ} / \mathrm{mol}-2 \Delta_{\mathrm{f}} G^{\mathrm{o}}$ glyceraldehyde $=-53 \mathrm{~kJ} / \mathrm{mol}$

$\Delta_{\mathrm{f}} G^{\mathrm{o}}$ " glyceraldehyde $=-187 \mathrm{~kJ} / \mathrm{mol}$

Individual Gibbs free energy of glyceraldehyde: $\mathrm{C}_{3} \mathrm{H}_{6} \mathrm{O}_{3}=\left(\mathrm{CH}_{2} \mathrm{O}\right)_{3}$

Known $\quad \Delta_{\mathrm{f}} G^{\mathrm{o}}$ glyceraldehyde $-3 \Delta_{\mathrm{f}} G^{\mathrm{o}}{ }_{\mathrm{H} 2}=\Delta_{\mathrm{f}} G^{\mathrm{o}}$ glyceraldehyde $=G^{\mathrm{o}}$ glyceraldehyde (the unknown) $-3 G^{\mathrm{o}}{ }_{\mathrm{H}_{2}}-1.5 G^{\mathrm{o}} \mathrm{O}_{2}-3 G^{\mathrm{o}} \mathrm{C}$

In $\mathrm{kJ} / \mathrm{mol}: \quad-187-3 \times 82 \mathrm{~kJ} / \mathrm{mol}=G_{\text {glyceraldehyde }}^{\mathrm{o}}-3(-406.5)-1.5(-463.5)-3(-671)$

Left-hand side: $-187 \mathrm{~kJ} / \mathrm{mol}-3 \times 82 \mathrm{~kJ} / \mathrm{mol}=\mathbf{- 4 3 0} \mathbf{~ k J} / \mathbf{m o l} \approx \Delta_{\mathrm{f}} G_{\text {glyceraldehyde }}^{\mathrm{o}}$ close to the estimate above.

$-430 \mathrm{~kJ} / \mathrm{mol}=G^{\mathrm{o}}$ glyceraldehyde $+3928 \mathrm{~kJ} / \mathrm{mol}$

$G_{\text {glyceraldehyde }}^{\mathrm{o}}=-4358 \mathrm{~kJ} / \mathrm{mol}$; average per bond: $/ 12=-363 \mathrm{~kJ} / \mathrm{mol}$; per 2 bonds: $\times 2=\underline{-727 \mathrm{~kJ} / \mathrm{mol}}$ per 2 bonds 
Conventional vs. meaningful individual free energies of ions. The traditional energetics of half reactions, while producing the right results for redox reaction pairs, are in themselves extremely hard to interpret, in particular if ions are involved. We demonstrate this for the half reaction of

$$
\mathbf{F e}^{3+}(\mathrm{aq})+\mathrm{e}^{-} \rightarrow \mathbf{F e}^{2+}(\mathrm{aq}) \quad E_{\text {red }}^{\mathrm{o}}=+0.77 \mathrm{~V}, \quad \Delta_{\mathrm{r}} G^{\mathrm{o}}=-74.2 \mathrm{~kJ} / \mathrm{mol}
$$

It easy to misinterpret the difference between the free energies of formation, $-78.9 \mathrm{~kJ} / \mathrm{mol}-(-4.7 \mathrm{~kJ} / \mathrm{mol})=$ $-74.2 \mathrm{~kJ} / \mathrm{mol}$, as the difference in the energies of these species. Unfortunately, unlike with the free-atomreferenced individual quantities $G_{\mathrm{Fe}^{3}}^{\mathrm{o}}$ etc., the difference of $-74.2 \mathrm{~kJ} / \mathrm{mol}$ does not compare the free energies of $\mathbf{F e}^{2+}(\mathrm{aq})$ vs. $\mathbf{F e}^{3+}$ (aq). Instead, $-74.2 \mathrm{~kJ} / \mathrm{mol}$ is the free energy change of the reaction

$$
\mathbf{F e}^{2+}(\mathrm{aq})+\mathrm{H}^{+}(\mathrm{aq}) \rightarrow \mathbf{F e}^{3+}(\mathrm{aq})+1 / 2 \mathrm{H}_{2}
$$

("relative to the standard hydrogen electrode"), which is rather difficult to interpret due to the other species $\left(\mathrm{H}^{+}(\mathrm{aq})\right.$ and $\left.\mathrm{H}_{2}\right)$ showing up. As we will show below, $\mathrm{H}^{+}(\mathrm{aq})$, requiring energy input for the ionization, is a fairly high-energy species $\left(G_{\mathrm{H}^{+}}^{\mathrm{o}}=+210 \mathrm{~kJ} / \mathrm{mol}\right.$ at $\left.\mathrm{pH} 0\right)$, while $\mathrm{H}_{2}$ is of low free energy $\left(G_{\mathrm{H}_{2}}^{\mathrm{o}}=-407\right.$ $\mathrm{kJ} / \mathrm{mol}$ ), being stabilized by a fairly strong bond. The energy difference that these properties of hydrogen introduce,

$$
1 / 2(-407 \mathrm{~kJ} / \mathrm{mol})-210 \mathrm{~kJ} / \mathrm{mol}=-413 \mathrm{~kJ} / \mathrm{mol} \text {, }
$$

is the shift between the conventional, standard-hydrogen electrode referenced, and the meaningful freeatom-referenced scale for $\Delta_{\mathrm{r}} G^{\mathrm{o}}$. The meaningful free-energy values $G^{\mathrm{o}}$ i reflect bond or cohesive energies and ionization energies in water or other solvation environments. We have found (see Table S1) $G^{\mathrm{o}} \mathrm{Fe}^{2+}=368 \mathrm{~kJ} / \mathrm{mol}$ and $G^{\mathrm{o}} \mathrm{Fe}^{3+}=856 \mathrm{~kJ} / \mathrm{mol}$, different by $413 \mathrm{~kJ} / \mathrm{mol}+74.2 \mathrm{~kJ} / \mathrm{mol}$.

Evaluating free energies of reactants and products. In the main text, the calculation of $\Delta_{\mathrm{r}} G^{\mathrm{o}}$ from free energies of reactants and products was mentioned as the first (i) of two approaches. This can be performed using conventional free energies of formation or meaningful individual free-energy values. We demonstrate the calculation for the reaction

$$
\mathrm{O}_{2}+4 \mathrm{H}^{+}(\mathrm{aq})+4 \mathrm{Fe}^{2+}(\mathrm{aq}) \rightarrow 2 \mathrm{H}_{2} \mathrm{O}+4 \mathrm{Fe}^{3+}(\mathrm{aq})
$$

For conceptual simplicity, we consider ferrous and ferric ions in water, rather than in cytochrome $c$.

(ia) Conventionally, one uses the Gibbs free energies of formation, $\Delta_{\mathrm{f}} G^{\mathrm{o}}$,

$$
\begin{aligned}
& \Delta_{\mathrm{r}} G^{\mathrm{o}}=\sum \nu_{\mathrm{i}} \Delta_{\mathrm{f}} G_{\text {prod, } \mathrm{i}}^{\mathrm{o}}-\sum \nu_{\mathrm{i}} \Delta_{\mathrm{f}} G_{\text {reactants, } \mathrm{i}}^{\mathrm{o}} \\
& \left.=\left(2 \Delta_{\mathrm{f}} G_{\mathrm{H}_{2} \mathrm{O}}^{\mathrm{o}}+4 \Delta_{\mathrm{f}} G^{\mathrm{o}} \mathrm{Fe}^{3+}(\mathrm{aq})\right)-\left(\Delta_{\mathrm{f}} G^{\mathrm{o}} \mathrm{O}_{2}+4 \Delta_{\mathrm{f}} G^{\mathrm{o}}{ }_{\mathrm{H}}^{+}+4 \Delta_{\mathrm{f}} G^{\mathrm{o}}{ }_{\mathrm{Fe}^{2+}}{ }_{(\mathrm{aq}}\right)\right) \\
& =2(-237)+4 \times(-5) \quad-(0+0+4 \times(-79))=-178 \mathrm{~kJ} / \mathrm{mol}
\end{aligned}
$$

The result is accurate but the $\Delta_{\mathrm{f}} G_{\mathrm{i}}^{\mathrm{o}}$ values cannot be interpreted in simple terms. 


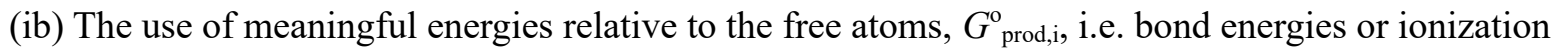
energies in water, provides more insight. Here, the calculation is

$$
\begin{aligned}
& \Delta_{\mathrm{r}} G^{\mathrm{o}}=G_{\text {prod }}^{\mathrm{o}}-G^{\mathrm{o}} \text { reactants }=\sum \nu_{\mathrm{i}} G_{\text {prod,i }}^{\mathrm{o}}-\sum \nu_{\mathrm{i}} G_{\text {reactants, } \mathrm{i}}^{\mathrm{o}} \\
& =\left(2 G_{\mathrm{H} 2 \mathrm{O}}^{\mathrm{o}}+4 G_{\mathrm{Fe}^{3+}(\mathrm{aq})}^{\mathrm{o}}\right)-\left(G_{\mathrm{O} 2}^{\mathrm{o}}+4 G_{\mathrm{H}^{+}}^{\mathrm{o}}+4 G_{\mathrm{Fe}^{2+}(\mathrm{aq})}^{\mathrm{o}}\right) \\
& =[2(-876)+4 \times 856-(-464+4 \times 210+4 \times 368)] \mathrm{kJ} / \mathrm{mol}=-176 \mathrm{~kJ} / \mathrm{mol}
\end{aligned}
$$

Every term has a meaning here: Bonding stabilizes $\mathrm{H}_{2} \mathrm{O}$ by $-876 \mathrm{~kJ} / \mathrm{mol}$ and $\mathrm{O}_{2}$ by $-464 \mathrm{~kJ} / \mathrm{mol}$, while the ionization free energy in water ${ }^{7}$ is $856 \mathrm{~kJ} / \mathrm{mol}$ for $\mathrm{Fe}^{3+}, 368 \mathrm{~kJ} / \mathrm{mol}$ for $\mathrm{Fe}^{2+}$, and $210 \mathrm{~kJ} / \mathrm{mol}$ for $\mathrm{H}^{+}$at $\mathrm{pH} 0$ (it would be $170 \mathrm{~kJ} / \mathrm{mol}$ at $\mathrm{pH} 7$ ).

"Weak bonds to strong bonds" applies only with a constant number of bonds. Chemical energy is released when weak bonds are broken and stronger bonds form. However, it must be remembered that this analysis implies an unchanged number of bonds. As an example, consider

$\begin{array}{lcccc} & \mathrm{C}_{6} \mathrm{H}_{12} \mathrm{O}_{6} \rightarrow 3 \mathrm{CO}_{2}+3 \mathrm{CH}_{4} \quad \Delta_{\mathrm{r}} H^{\mathrm{o}}=-132 \mathrm{~kJ} / \mathrm{mol}, \Delta_{\mathrm{r}} G^{\mathrm{o}}=-430 \mathrm{~kJ} / \mathrm{mol} \\ \text { Average bond energy }(\mathrm{kJ} / \mathrm{mol}): & -403 & \mathbf{- 8 0 4} & -415 \\ \text { Average bond free energy: } & -365 & -\mathbf{7 6 4} & -384 \\ \text { Conventional bond count: } & 24 & 6 & 12 \text { decrease in number of bonds }\end{array}$

Very strong $\mathrm{C}=\mathrm{O}$ bonds in $\mathrm{CO}_{2}$ (with a $\mathrm{C}=\mathrm{O}$ bond energy of $804 \mathrm{~kJ} / \mathrm{mol}$ ) are formed, so a naïve analysis would predict a highly exothermic reaction. However, this prediction is incorrect. It fails because only relatively few of those strong bonds have been formed. If a double bond is counted as one bond, the number of bonds is reduced by six. A meaningful general analysis can be made most easily if the number of bonds remains unchanged after the reaction, which is the case for electron-pair bonds. ${ }^{1}$ This means that a double bond needs to be counted as two, and a triple bond as three bonds:

Electron-pair bonds:

$$
\mathrm{C}_{6} \mathrm{H}_{12} \mathrm{O}_{6} \rightarrow 3 \mathrm{CO}_{2}+3 \mathrm{CH}_{4} \quad \Delta_{\mathrm{r}} H^{\mathrm{o}}=-132 \mathrm{~kJ} / \mathrm{mol}, \Delta_{\mathrm{r}} G^{\mathrm{o}}=-430 \mathrm{~kJ} / \mathrm{mol}
$$

Per two electron-pair bonds:

$\begin{array}{llll}H^{\mathrm{o}}(\mathrm{kJ} / \mathrm{mol}) & -806 & -804 & -830 \\ G^{\mathrm{o}}(\mathrm{kJ} / \mathrm{mol}) & -730 & -764 & -768\end{array}$

The small difference in the energies of electron-pair bonds in reactants and products immediately shows that the reaction will be only mildly exothermic or exergonic.

The small entropy of combustion. Our previous quantitative analysis ${ }^{1}$ focused on $\Delta_{\mathrm{c}} H^{\mathrm{o}}$, the enthalpy of combustion, which equals the heat evolved in an uncontrolled reaction with $\mathrm{O}_{2}$ at constant $T$ and $P$. In respiration, we are interested in the free energy change of reactions with $\mathrm{O}_{2}$, which can be used to pump protons and eventually generate ATP. To evaluate $\Delta_{c} G^{\mathrm{o}}=\Delta_{\mathrm{c}} H^{\mathrm{o}}-T \Delta_{\mathrm{c}} S^{\mathrm{o}}$ we need for a general combustion of organic molecules 


$$
\begin{aligned}
& \mathrm{C}_{c} \mathrm{O}_{o} \mathrm{H}_{h} \mathrm{~N}_{n}+v_{\mathrm{O}_{2}} \mathrm{O}_{2} \rightarrow v_{\mathrm{H}_{2} \mathrm{O}} \mathrm{H}_{2} \mathrm{O}(l)+v_{\mathrm{CO}_{2}} \mathrm{CO}_{2}+v_{\mathrm{N}_{2}} \mathrm{~N}_{2} \\
& \begin{aligned}
\Delta_{c} S^{\mathrm{o}} \approx v_{\mathrm{H}_{2} \mathrm{O}}\left(S_{\mathrm{H}_{2} \mathrm{O}}=70 \mathrm{~J} / \mathrm{molK}\right)+v_{\mathrm{CO}_{2}}\left(S_{\mathrm{CO}_{2}}=214 \mathrm{~J} / \mathrm{molK}\right)+v_{\mathrm{N}_{2}}\left(S_{\mathrm{N}_{2}}=192 \mathrm{~J} / \mathrm{molK}\right) \\
\quad-v_{\mathrm{O}_{2}}\left(S_{\mathrm{O}_{2}}=205 \mathrm{~J} / \mathrm{molK}\right)-S_{\text {fuel }} \\
\\
\approx v_{\mathrm{H}_{2} \mathrm{O}} 70 \mathrm{~J} / \mathrm{molK}+\left(v_{\mathrm{CO}_{2}}-v_{\mathrm{O}_{2}}+v_{\mathrm{N}_{2}}\right) 205 \mathrm{~J} / \mathrm{molK}-S_{\text {fuel }} \\
\quad=(h / 3+c-c-h / 4+o / 2+n / 2) 205 \mathrm{~J} / \mathrm{molK}-S_{\text {fuel }} \\
\quad=(o+n) 103 \mathrm{~J} / \mathrm{molK}+h 20 \mathrm{~J} / \mathrm{molK}-S_{\text {fuel }}
\end{aligned}
\end{aligned}
$$

Since both reactants and products contain similar numbers of linear gas molecules, there is significant cancelation. The entropic contribution to the free energy of combustion at the standard temperature of $T=$ $298 \mathrm{~K}$ is

$$
-T \Delta_{\mathrm{c}} S^{\mathrm{o}} \approx(o+n)(-30 \mathrm{~kJ} / \mathrm{mol})+h 6 \mathrm{~kJ} / \mathrm{mol}+298 \mathrm{~K} S_{\text {fuel }}^{\mathrm{o}}
$$

Since the last term partially cancels the second, this is usually a fairly small correction to ${ }^{1}$

$$
\Delta_{\mathrm{c}} H^{\mathrm{o}}=(c+0.3 h-0.5 o)(-418 \mathrm{~kJ} / \mathrm{mol})
$$

which applies well if $c \geq o+n$. Overall, we obtain

$$
\begin{aligned}
\Delta_{\mathrm{c}} G^{\mathrm{o}} & =\Delta_{\mathrm{c}} H^{\mathrm{o}}-T \Delta_{\mathrm{c}} S^{\mathrm{o}} \\
& \approx(c+0.314 h-0.43 o+0.07 n)(-418 \mathrm{~kJ} / \mathrm{mol})+298 \mathrm{~K} S_{\text {fuel }}^{\mathrm{o}}
\end{aligned}
$$

"Reduced molecules". A common misconception attributes high energy to "reduced molecules", ${ }^{11}$ see also ref. $^{12}$, p.529 and p. 662, top. To start with, the terminology is confused. The total oxidation number of an uncharged molecule is zero and therefore, unlike an ion, a molecule is rarely reduced. What changes is the oxidation state of carbon, so the terminology "reduced molecules" actually means "reduced-carbon molecules". This 'concept' is often closely tied to an electron-transfer picture that ignores that oxidation numbers of covalently bonded atoms are mostly fictitious. For instance, in $\mathrm{H}_{2} \mathrm{O}$ the $\mathrm{O}$ atom does not have net +2 electrons, but only has an effective atomic charge of -0.66 (see Figure $\mathrm{S} 4$ below).

The supposed higher energy of reduced-carbon molecules is really the higher energy of the larger number of $\mathrm{O}_{2}$ molecules used in their combustion: Reduced-carbon molecules usually contain little $\mathrm{O}$; we have pointed out and shown quantitatively that molecules containing oxygen atoms have a lower heat of combustion per mass since they pull less of the energy-providing $\mathrm{O}_{2}$ into the reaction. ${ }^{1}$

This misconception is closely related to the faulty assumption that the heat of combustion derives from the fuel. In this context, it is useful to realize that $\mathrm{CH}_{4}$ has the same total bond energy as $\mathrm{CO}_{2}$ (actually, $\mathrm{CH}_{4}$ is slightly lower in energy (more stable) relative to the free atoms). ${ }^{1}$

The claim that reduced molecules are high-energy molecules has a further flaw: Without $\mathrm{O}_{2}$, partially oxidized molecules like glucose (carbohydrates) can actually release more energy than 
hydrocarbons as shown in the main text. Anaerobic respiration releases energy from less reduced glucose, but not from nearly fully reduced fatty acids.

Reaction energetics of reduced molecules. In the main text, reactions of $\mathrm{CH}_{4}$ were analyzed and shown to be endergonic unless $\mathrm{O}_{2}$ (or another strong oxidant) is involved. As an example of a slightly larger reduced molecule, we consider n-hexane, for which the needed thermodynamic data can be found fairly easily:

$$
\begin{array}{ll}
\mathrm{C}_{6} \mathrm{H}_{14} \rightarrow \mathrm{C}_{6} \mathrm{H}_{6}+4 \mathrm{H}_{2} & \Delta_{\mathrm{r}} G^{\mathrm{o}}=+128 \mathrm{~kJ} / \mathrm{mol} \\
\mathrm{C}_{6} \mathrm{H}_{14} \rightarrow 6 \mathrm{C}(\mathrm{s})+7 \mathrm{H}_{2} & \Delta_{\mathrm{r}} G^{\mathrm{o}}=+4 \mathrm{~kJ} / \mathrm{mol} \\
\mathrm{C}_{6} \mathrm{H}_{14}+12 \mathrm{H}_{2} \mathrm{O} \rightarrow 19 \mathrm{H}_{2}+6 \mathrm{CO}_{2} & \Delta_{\mathrm{r}} G^{\mathrm{o}}=+484 \mathrm{~kJ} / \mathrm{mol} \\
\mathrm{C}_{6} \mathrm{H}_{14}+6 \mathrm{H}_{2} \mathrm{O} \rightarrow \mathrm{C}_{6} \mathrm{H}_{12} \mathrm{O}_{6}+7 \mathrm{H}_{2} & \Delta_{\mathrm{r}} G^{\mathrm{o}}=+515 \mathrm{~kJ} / \mathrm{mol} \\
\mathrm{C}_{6} \mathrm{H}_{14}+3 \mathrm{CO}_{2} \rightarrow \mathrm{C}_{6} \mathrm{H}_{12} \mathrm{O}_{6}+3 \mathrm{C}+\mathrm{H}_{2} & \Delta_{\mathrm{r}} G^{\mathrm{o}}=+275 \mathrm{~kJ} / \mathrm{mol} \\
\mathrm{C}_{6} \mathrm{H}_{14}+5 \mathrm{H}_{2} \rightarrow 6 \mathrm{CH}_{4} & \Delta_{\mathrm{r}} G^{\mathrm{o}}=-302 \mathrm{~kJ} / \mathrm{mol} \\
\mathrm{C}_{6} \mathrm{H}_{14}+5 \mathrm{H}_{2} \mathrm{O} \rightarrow 6 \mathrm{CH}_{4}+2.5 \mathrm{O}_{2} & \Delta_{\mathrm{r}} G^{\mathrm{o}}=+883 \mathrm{~kJ} / \mathrm{mol}
\end{array}
$$

We can also analyze the decomposition of palmitate with water to $\mathrm{NADPH}$ and $\mathrm{CO}_{2}$ :

$$
\begin{aligned}
& \mathrm{C}_{15} \mathrm{H}_{31} \mathrm{COOH}+30 \mathrm{H}_{2} \mathrm{O}+46 \mathrm{NADP}^{+} \rightarrow 46\left(\mathrm{NADPH}+\mathrm{H}^{+}\right)+16 \mathrm{CO}_{2} \\
& -305\left(\text { ref. }^{9}\right) \quad 30(-237) \quad 46(-17) \quad 16(-394) \quad \Delta_{\mathrm{r}} G^{\mathrm{o}^{\prime}}=+329 \mathrm{~kJ} / \mathrm{mol}
\end{aligned}
$$

Most of these reactions are endergonic, confirming that reduced molecules are low-energy species. Only in combustion do they release the energy stored in the oxidant, i.e. in $\mathrm{O}_{2}$, particularly efficiently.

$\mathrm{NAD}(\mathbf{P}) \mathbf{H}+\mathbf{H}^{+}$is analogous to $\mathbf{H}_{2}$. A useful shortcut in bioenergetic calculations is provided by considering $\mathrm{NAD}(\mathrm{P}) \mathrm{H}+\mathrm{H}^{+}$as a biological analogue of $\mathrm{H}_{2}$, performing the same reductive hydrogen transfer with very similar free energy changes. For instance, consider the protonation of

$$
\text { fumarate, }{ }^{-} \mathrm{OOC}-\mathrm{CH}=\mathrm{CH}_{-} \mathrm{COO}^{-}, \quad \text { to succinate, }{ }^{-} \mathrm{OOC}-\mathrm{CH}_{2}-\mathrm{CH}_{2}-\mathrm{COO}^{-}
$$

$$
\begin{array}{lrlrl}
\text { Fumarate }+\mathbf{N A D}(\mathbf{P}) \mathbf{H}+\mathbf{H}^{+} & \rightarrow \mathbf{N A D}(\mathbf{P})^{+}+\text {succinate } & \Delta_{\mathrm{r}} G^{\mathrm{o}^{\prime}}=-113 \mathrm{~kJ} / \mathrm{mol} \\
\text { Fumarate }+ & \mathbf{H}_{2} & \rightarrow \quad \text { succinate } & \Delta_{\mathrm{r}} G^{\mathrm{o}^{\prime}}=-130 \mathrm{~kJ} / \mathrm{mol}
\end{array}
$$

or of

$$
\begin{array}{lccc}
\text { coenzyme Q (ubiquinone) } & \text { to ubiquinol, } \mathrm{QH}_{2}: \\
\mathrm{Q}+\mathbf{N A D}(\mathbf{P}) \mathbf{H}+\mathbf{H}^{+} \rightarrow \mathbf{N A D}(\mathbf{P})^{+}+\mathrm{QH}_{2} \Delta_{\mathrm{r}} G^{\mathrm{o}^{\prime}}=-81 \mathrm{~kJ} / \mathrm{mol} \\
\mathrm{Q}+\quad \mathbf{H}_{2} \rightarrow & \mathrm{QH}_{2} & \Delta_{\mathrm{r}} G^{\mathrm{o}^{\prime}}=-98 \mathrm{~kJ} / \mathrm{mol}
\end{array}
$$

The small 17-kJ/mol difference in free energies of reaction is systematic:

$$
\mathrm{NAD}(\mathrm{P}) \mathrm{H}+\mathrm{H}^{+} \rightarrow \mathrm{NAD}(\mathrm{P})^{+}+\mathrm{H}_{2} \quad \Delta_{\mathrm{r}} G^{\mathrm{o}^{\prime}}=+17 \mathrm{~kJ} / \mathrm{mol}(-0.09 \mathrm{~V})
$$


For analyzing the energetics within a biochemical reaction we can replace

$$
\mathrm{NAD}(\mathrm{P})-\mathrm{H}+\mathrm{H}^{+} \rightarrow \mathrm{NAD}(\mathrm{P})^{+}+
$$

with

"H"-H + $\mathrm{H}^{+} \rightarrow$ "H"

and even shorter

$$
\text { "H } \mathrm{H}_{2} \text { NAD(P)H } \rightarrow
$$

So we can treat " $\mathrm{H}_{2}$ " $\mathrm{NAD}(\mathrm{P}) \mathrm{H}$ as a hypothetical form of $\mathrm{H}_{2}$ with $\Delta_{\mathrm{f}} G^{0}$ " $\mathrm{H} 2$ " $(\mathrm{NAD}(\mathrm{P}) \mathrm{H})=-17 \mathrm{~kJ} / \mathrm{mol}$, or $\Delta_{\mathrm{f}} G^{\mathrm{o}}$ " $\mathrm{H} 2$ " $(\mathrm{NAD}(\mathrm{P}) \mathrm{H})=+61 \mathrm{~kJ} / \mathrm{mol}$ (which is at $-21 \mathrm{~kJ} / \mathrm{mol}$ relative to $\left.\mathrm{H}_{2}(\mathrm{~g})\right)^{13,14}$.

Consider, for instance, $\quad \mathrm{CO}_{2}+2\left(\mathrm{NADPH}+\mathrm{H}^{+}\right) \rightarrow \mathrm{CH}_{2} \mathrm{O}+\mathrm{H}_{2} \mathrm{O}+\mathrm{NADP}^{+}$

The simplified reaction is $\quad \mathrm{CO}_{2}+2$ " $\mathrm{H}_{2}$ " ${ }_{\mathrm{NAD}(\mathrm{P}) \mathrm{H}} \rightarrow \mathrm{CH}_{2} \mathrm{O}+\mathrm{H}_{2} \mathrm{O}$

which has an only slightly $(17 \mathrm{~kJ} / \mathrm{mol})$ higher $\Delta_{\mathrm{r}} G^{\mathrm{o}}$ than the reaction with real $\mathrm{H}_{2}$.

$\mathrm{O}_{2}$ in the last or first step of respiration? In the conventional presentation, the reaction with $\mathrm{O}_{2}$ is usually shown as the last, and correspondingly $\mathrm{O}_{2}$ is termed the terminal electron acceptor. Based on Figure $\mathrm{S} 1$, one can argue that the reaction with $\mathrm{O}_{2}$ is not the last but the first in the chain. A reaction naturally proceeds from high-energy reactants to lower-energy products, so the order cannot be switched. As shown in Figure $\mathrm{S} 1 \mathrm{~b}$, even if steps are left out, $\mathrm{O}_{2}$ is still among the high-energy reactants, which must be present in the beginning for the series of reactions to start. That most of the energy released in respiration can be attributed to $\mathrm{O}_{2}$ becomes clear when comparing with the corresponding reaction without $\mathrm{O}_{2}$ (and forming $\mathrm{H}_{2}$ instead of $\mathrm{H}_{2} \mathrm{O}$ ), see Figure S1c.

It can be argued that in steady state, even the high-energy species such as $\mathrm{Fe}^{3+}$ and $\mathrm{Q}$ are present in significant concentration. Nevertheless, in such a steady state a "first" or a "terminal" reaction cannot be uniquely identified, and one can still choose the reaction with $\mathrm{O}_{2}$ as the first in the energy-transfer chain.

Presenting the electron transfer chain of aerobic respiration correctly. The relevance of the analysis of the electron transfer chain in this paper is not based on every step occurring as claimed, or with an exact free-energy change. Rather, the goal is to show how a series of electron-transfer processes can be described in a proper redox energy transfer and release analysis. If details of the electron-transfer process are modified, the energy analysis can be adjusted accordingly. Some of the species in the "electron transport" chains are separated by only nanometers and certain oxidation states are extremely short-lived. Their analysis, like that of radicals in combustion, does not shed light on the bioenergetics framework, which is the focus of this analysis. The interest here is in the overall energetics, not individual reaction mechanisms.

The chemical equations reviewed in the main text show that electrons are transferred through the following species:

\section{$\mathrm{NADH} \rightarrow \mathrm{QH}_{2} \rightarrow 2 \mathrm{Fe}^{2+}(\mathrm{cyt} c) \rightarrow \mathrm{H}_{2} \mathrm{O}$}


Note that all of these are donor (i.e. reduced) species. These species ferry the electrons to the next reaction site. Some representations of electron transport chains incorrectly show some acceptors, in particular in the last step where $\mathrm{O}_{2}$ is involved (furthermore without its required reaction partner, $\mathrm{H}^{+}$, which is the main electron acceptor, see the main text and Figure S4 below). ${ }^{12}$

Why should there be no acceptors in the electron-transfer chain? An acceptor is ready to accept electrons it does not have. As soon as it receives the electron, it stops being an acceptor, having become a donor for the next step. So an acceptor never contains the transferred electron and must not be shown in a simple electron transport chain.

An expanded representation that shows all species involved, in particular $\mathrm{O}_{2}$, could be constructed as follows:

$$
\begin{aligned}
\mathbf{N A D H} / \mathrm{NAD}^{+}+\mathrm{H}^{+}(\mathrm{aq}) & \rightarrow \mathrm{Q}+2 \mathrm{H}^{+}(\mathrm{aq}) / \mathbf{Q H} / \mathrm{Q}+2 \mathrm{H}^{+}(\mathrm{aq}) \\
& \rightarrow 2 \mathrm{Fe}^{3+}(\mathrm{cyt} c) / \mathbf{2} \mathbf{F e}^{2+}(\mathbf{c y t} \boldsymbol{c}) / 2 \mathrm{Fe}^{3+}(\text { cytc }) \rightarrow 1 / 2 \mathrm{O}_{2}+2 \mathrm{H}^{+}(\mathrm{aq}) / \mathbf{H}_{2} \mathbf{O}
\end{aligned}
$$

Bold font labels the species containing the transferred electron. Note that just showing donor/acceptor pairs, e.g. $\rightarrow \mathbf{Q H}_{2} / \mathrm{Q} \rightarrow$ does not properly represent the process, which includes a reaction with $\mathrm{Q}+2$ $\mathrm{H}^{+}(\mathrm{aq})$ as products and a subsequent one with $\mathrm{Q}+2 \mathrm{H}^{+}(\mathrm{aq})$ as reactants.

Finally, it has to be acknowledged that it may also be misleading to refer to $\mathrm{H}_{2} \mathrm{O}$ simply as an electron donor. $\mathrm{H}_{2} \mathrm{O}$ does not donate an electron to become $\mathrm{H}_{2} \mathrm{O}^{+}$. While for $\mathrm{Fe}^{2+}(\mathrm{aq}) \rightarrow \mathrm{Fe}^{3+}(\mathrm{aq})+\mathrm{e}^{-}$, we can indeed consider just the electron-release or ionization energy in water, for a molecule like $\mathrm{H}_{2} \mathrm{O}$ that splits up and recombines into ions and a species with very different bonding, $\mathrm{O}=\mathrm{O}$, bond-energy changes are more crucial energetically than is electron release.

Different views of energy release in the electron transfer chain of aerobic respiration. The following Figures S1-S3 show different representation of the sequence of reactions in aerobic respiration: The "electron waterfall" in Figure S2, which does not show the energies of reactants and products, and a true energy-level diagram that does, Figure S1, but is hampered by complexity. The redox energy transfer and release (RETAR) diagram shown in Figure 2c of the main text is superior in showing the origin of chemical energy in these reactions. Potential variants of the RETAR diagram are explained in Figure S3. 
(a)

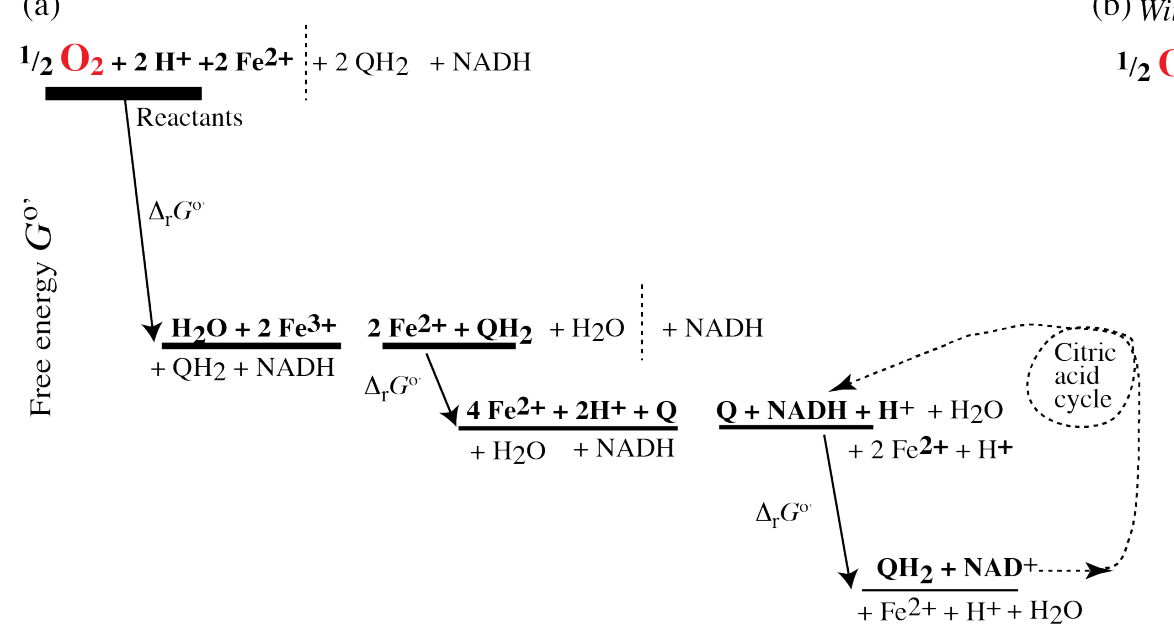

(b) Without $\mathrm{Fe}^{2+}$ or $\mathrm{QH}_{2}$ :

(c) Without $\mathrm{O}_{2}$ :

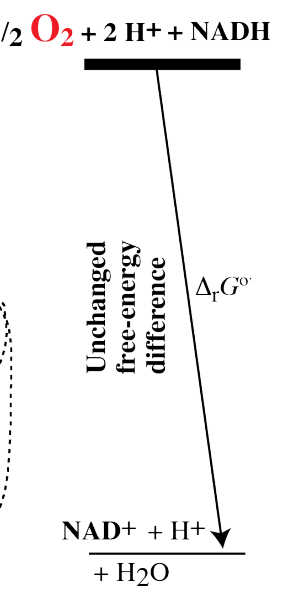

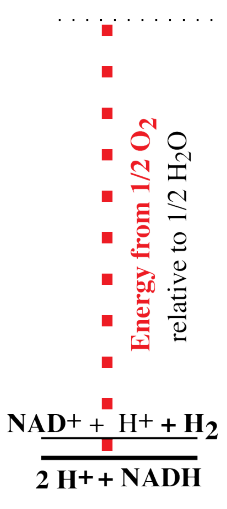

Figure S1. (a) The free-energy level approach described in the context of eq.(15), applied (somewhat inconveniently) to a series of reactions in the 'terminal' electron transfer chain of aerobic respiration. Note

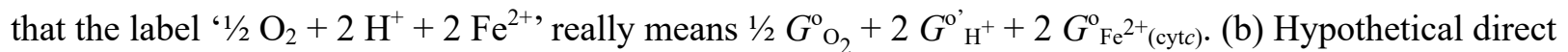
reaction of $1 / 2 \mathrm{O}_{2}+2 \mathrm{H}^{+}$with $\mathrm{NADH}$, without $2 \mathrm{Fe}^{2+}$ or $\mathrm{QH}_{2}$, after a suitable vertical shift shown for reference. The total free energy change of reaction is the same as for the series of reactions in part a. (c) Energetics of the half reaction $2 \mathrm{H}^{+}(\mathrm{aq})+2 \mathrm{e}^{-} \rightarrow \mathrm{H}_{2}(\mathrm{~g})$ at $\mathrm{pH}$, after a suitable vertical shift, shown for reference to highlight the crucial effect of $\mathrm{O}_{2}$.

"Half-reaction free-energy levels". In a correct free-energy diagram of a series of reactions as shown in Figure S1, it is inconvenient that reactants converted in later steps must be included in the analysis from the start. This is avoided in an alternative analysis in terms of half reactions, which is convenient for displaying a series of reactions, since the same half reaction (in reverse direction) can be used in subsequent reactions, see Figure S2. This is the currently accepted representation of the energetics of electron transfer chains. However, as pointed out in the main text, the levels do not correspond to energies of specific species and therefore do not reveal where chemical energy is stored.

In the main text it was pointed out that acceptors of high energy lower their half-reaction energy level. To make this point graphically, in Figure $\mathrm{S} 1 \mathrm{~b}$ the level for $\mathrm{F}_{2}+2 \mathrm{e}^{-} \rightarrow 2 \mathrm{~F}^{-}$is also shown; it is lower than all others. $F_{2}$ is undoubtedly a high-energy molecule due to its weak bond (bond formation enthalpy of $-155 \mathrm{~kJ} / \mathrm{mol}$ ). This confirms that "free-energy levels" associated with high-energy oxidants show up low in this plot of $\Delta_{\mathrm{red}} G^{\mathrm{o}}$.

Free energies of half reactions and standard reduction potentials. In the main text, it was derived, eq.(18a), that

$$
\Delta_{\mathrm{r}} G^{\mathrm{o}}=\Delta_{\mathrm{red}} G^{\mathrm{o}}{ }_{1}-\Delta_{\mathrm{red}} G_{2}^{\mathrm{o}}=\Delta \Delta_{\mathrm{red}} G^{\mathrm{o}}
$$

This equation may be more familiar in terms of standard reduction potentials (in volts), with the "standard cell voltage" $E^{\mathrm{o}}$

$$
E^{\mathrm{o}}=E_{\text {red,1 }}^{\mathrm{o}}+E_{\text {ox }, 2}^{\mathrm{o}}=E_{\text {red, } 1}^{\mathrm{o}}-E_{\text {red,2 }}^{\mathrm{o}}
$$


These equations are related by $\Delta_{\text {red }} G^{\circ}{ }_{i}=-v_{\mathrm{e}} F E_{\text {red,i }}^{\mathrm{o}}+v_{\mathrm{e}} c$. The constant $c$, which has the value of -413 $\mathrm{kJ} / \mathrm{mol}$ when meaningful bond energies and ionization free energies in water are used, ${ }^{7,15}$ is always subtracted out in measurable quantities such as the free energies of full redox reactions (see. eq(17a)) and is therefore of little consequence.

(a)

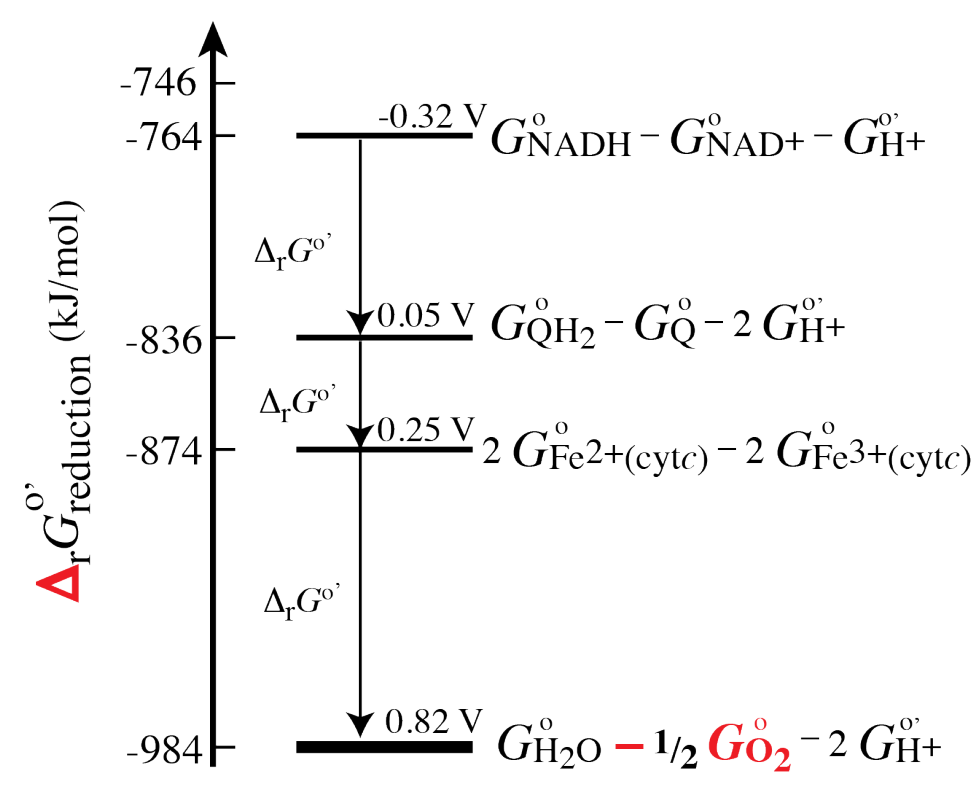

(b)

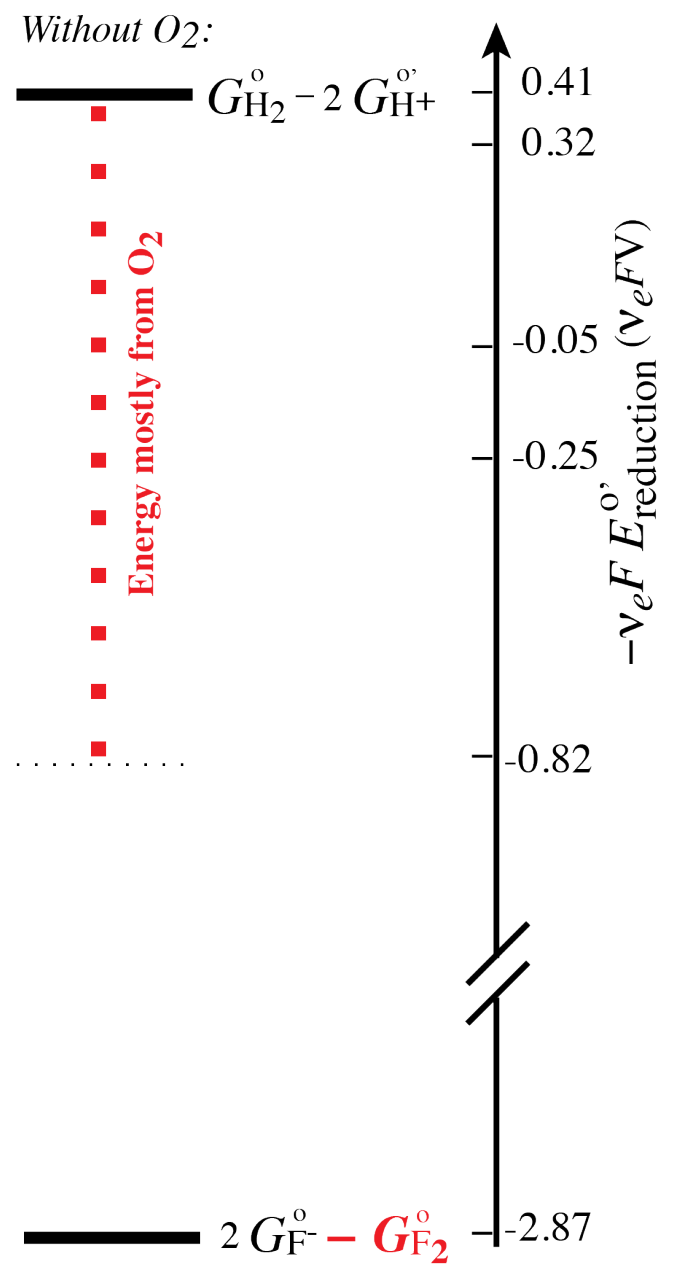

Figure S2. (a) Sequence of half-reaction energy levels in aerobic respiration ("electron waterfall"). Note that the arrows between the energy levels do not point from reactants to products. (b) Half reaction energy levels of two reactions without $\mathrm{O}_{2}$ shown for reference. At the top, the high level for $2 \mathrm{H}^{+}(\mathrm{aq})+2 \mathrm{e}^{-} \rightarrow \mathrm{H}_{2}$ (g) highlights the crucial effect of $\mathrm{O}_{2}$ (compare part a). At the bottom, the level of $\mathrm{F}_{2}(\mathrm{~g})+2 \mathrm{e}^{-} \rightarrow 2 \mathrm{~F}^{-}(\mathrm{aq})$ is shown, confirming that half reactions with high-energy, weakly bonded electron acceptors, in this case $\mathrm{F}_{2}$, show up low. On the right side of the diagram, the numerical values of the conventional standard reduction potentials (in volt) of the half reactions are shown for reference. 

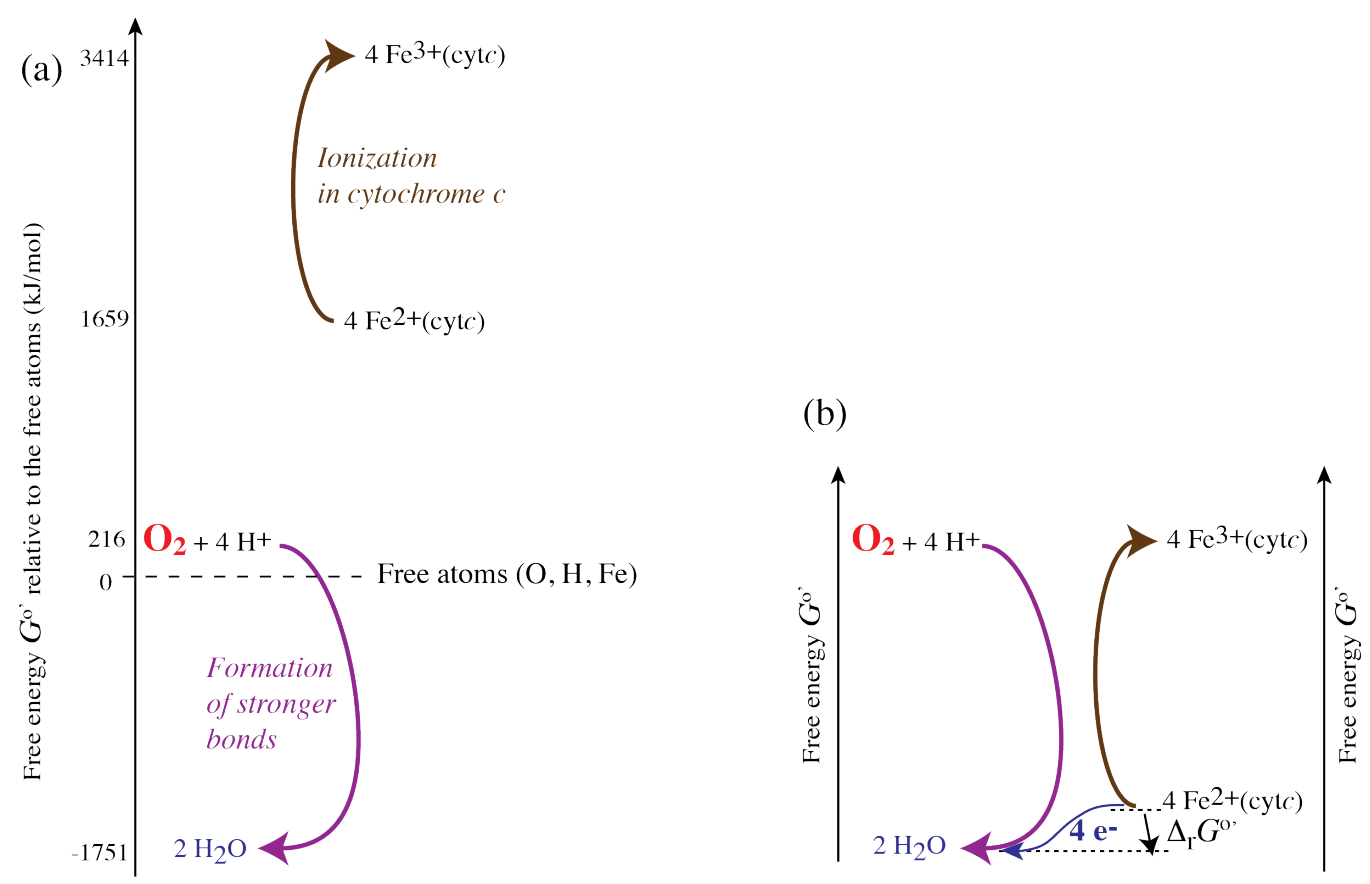

Figure S3. Common vertical shifts of the free energies of reactants and products in each half reaction. (a) Half reactions of reaction (14) on the natural free-energy scale relative to free atoms at $0 \mathrm{~kJ} / \mathrm{mol}$. (b) Same as in part a) after the high-energy species in the half reactions (top levels) have been aligned vertically, making the free energy change of the combined reaction, $\Delta_{\mathrm{r}} G^{\mathrm{o}^{\prime}}$, easy to read off at the bottom right. The electron transfer is also indicated.

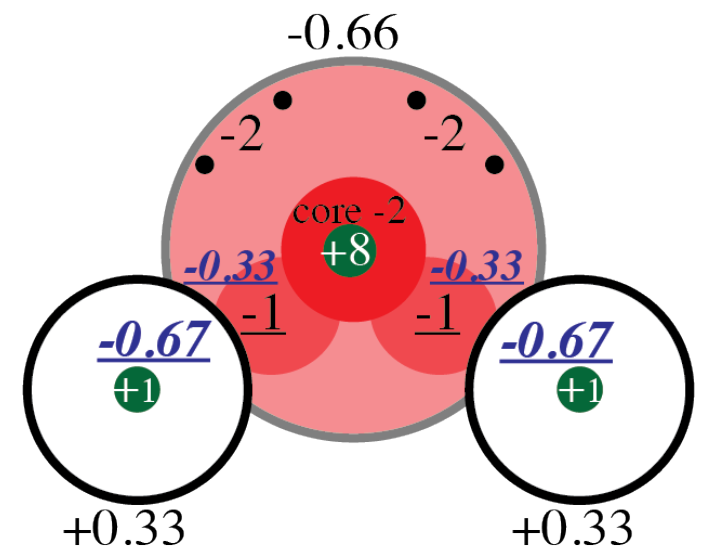

Figure S4. Schematic sketch of a water molecule with its nuclear charges, effective atomic charges (shown outside the molecule), and distribution of 10 electrons, in units of the elementary charge $e$. The charge contributions of the two electrons taken up by the molecule in half reaction (16a) are shown in blue italics. The two pairs of bonding electrons are underlined. Hydrogen's effective atomic charge of $+0.33 e$ means that $67 \%$ of an electron charge compensates the proton charge $(+1-0.67=+0.33)$ and is thus associated with hydrogen. Only $33 \%$ of the two electrons taken up by the molecule in half reaction (16a) are transferred to oxygen. 
Standard reduction potentials are not electron energies. Interpreting standard reduction potentials as electron free energies in a half-reaction or half-cell, which is the underpinning of the "electron waterfall" picture of electron transfer chains, is highly problematic. The energetics of electrons in a half-cell is a difficult problem. It requires consideration of the outer potential $\psi$, inner potential $\phi$, surface dipolar potential $\chi$, contact potential (difference) between different metals, work function $\Phi$, electrochemical potential, absolute electrode potential, etc. ${ }^{15}$ The energy of the electron in its surroundings (in the simplest case a metallic electrode) must be taken into account. As a simple model system, consider a $\mathrm{Zn} / \mathrm{Cu}$ galvanic cell:

$$
\begin{aligned}
& \mathrm{Zn}(\mathrm{s}) \rightarrow \mathrm{Zn}^{2+}(\mathrm{aq})+2 \mathrm{e}^{-}(\mathbf{Z n}) \\
& \mathrm{Cu}^{2+}(\mathrm{aq})+2 \mathrm{e}^{-}(\mathbf{C u}) \rightarrow \mathrm{Cu}(\mathrm{s})
\end{aligned}
$$

The free energy of the electron depends on the type of metal and on the (unmeasurable) inner (electrical) potential $\phi$. The energy of the electrons can be ignored only if the electrons end up in the same environment. Vacuum is such an environment, and for this reason one refers to vacuum absolute reduction potentials when only the difference of meaningful individual Gibbs free energies of chemical reactants and products, as we had done tacitly in the main text. This demonstrates that statements about the free energy of the electrons, e.g. in terms of standard reduction potentials, should be avoided. Fortunately, the electrons are only intermediates in the overall reaction and therefore their energies can be ignored. ${ }^{7}$

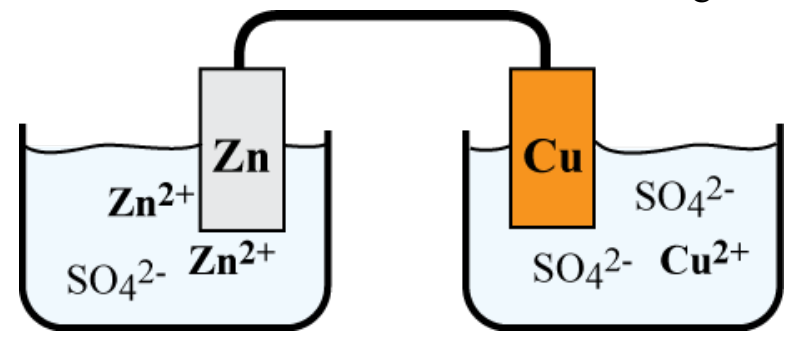

Figure S5. Model system with two half-cells of different standard reduction potentials where the electrons in the two electrodes have the same free energy (same electrochemical potential): $\mathrm{Zn} / \mathrm{Cu}$ galvanic cell with a good conductor connecting the electrodes, equalizing the electron electrochemical potential in the two electrodes, but without a salt bridge. (This set-up is not contrived but actually equivalent to two electrodes of two batteries connected in series, a common situation.)

Figure S5 shows a set-up where the electrons in the electrodes of two half-cells with different standard reduction potentials have the same free energy (are at the same electrochemical potential) because they are connected by a good conductor. This disproves the claim that the standard reduction potential is proportional to the electron free energy.

The electron waterfall picture appears to represent the electron energy, or electrochemical potential, in the various steps of the transfer process. This would necessarily refer to the electron energy in the donor species, since that is where the electron exclusively resides, as pointed out earlier. This, however, is easily shown to be incorrect. For instance, the energy level of an electron in $\mathrm{Fe}^{0}$ is not unique, being different for the half reactions $\mathrm{Fe}^{0} \rightarrow \mathrm{Fe}^{2+}+2 \mathrm{e}^{-}$and $\mathrm{Fe}^{0} \rightarrow \mathrm{Fe}^{3+}+3 \mathrm{e}^{-}$. 
Reduced "electron-carrying" molecules do not carry the energy. Ubiquinol, $\mathrm{QH}_{2}$, is a molecule in the electron-transfer chain that carries electrons (from complexes I and II to complex III). From the standard reduction potential $E^{\mathrm{o}^{\prime}}=0.045 \mathrm{~V}$, we can conclude $\mathrm{QH}_{2}$ is lower in free energy than $\mathrm{Q}+2 \mathrm{H}^{+}$by $-2 F E^{\mathrm{o}^{\prime}}-$ $2 \times 413 \mathrm{~kJ} / \mathrm{mol}=-835 \mathrm{~kJ} / \mathrm{mol}$, so $\mathrm{QH}_{2}$ is lower in energy than $\mathrm{Q}$ by $-835 \mathrm{~kJ} / \mathrm{mol}+2 \times 170 \mathrm{~kJ} / \mathrm{mol}=-495$ $\mathrm{kJ} / \mathrm{mol}$. This reduction in energy of $\mathrm{QH}_{2}$ relative to $\mathrm{Q}$ can be attributed to its stabilization by one extra $\mathrm{O}-\mathrm{H}$ bond: $\mathrm{Q}$ has four double bonds, $\mathrm{QH}_{2}$ three "double" bonds and two single $\mathrm{O}-\mathrm{H}$ bonds.

The analysis has shown that the electron-carrying, reduced form $\mathrm{QH}_{2}$ is of lower energy. Energy is carried by its oxidized counterpart, Q, which is less stabilized by bonding. When Q reacts with NADH + $\mathrm{H}^{+}$, some of this energy is released. On the other hand, when lower-energy $\mathrm{QH}_{2}$ reacts with a high-energy species, $\mathrm{Fe}^{3+}($ cytc), it gets charged up into its high-energy form, $\mathrm{Q}$.

Energetics of the Calvin cycle. The $\mathrm{CO}_{2}$-fixation reaction of photosynthesis is an important biochemical process with energy implications, which goes by various different names (dark reaction (fixation of $\mathrm{CO}_{2}$ ), Calvin(-Benson) cycle). The net effect of the cycle is that atmospheric $\mathrm{CO}_{2}$ elongates sugar molecules, with the needed hydrogen atoms (not energy) provided by $\mathrm{NADPH}+\mathrm{H}^{+}$produced in the primary reaction of photosynthesis, $\mathrm{H}_{2} \mathrm{O}+\mathrm{NADP}^{+} \rightarrow \mathrm{NADPH}+\mathrm{H}^{+}+1 / 2 \mathrm{O}_{2}$. The net reaction of the Calvin cycle is $3 \mathrm{CO}_{2}+6\left(\mathrm{NADPH}+\mathrm{H}^{+}\right)+9 \mathrm{ATP}+8 \mathrm{H}_{2} \mathrm{O} \rightarrow \mathrm{C}_{3} \mathrm{H}_{5} \mathrm{O}_{3} \mathrm{PO}_{3} \mathrm{H}+3 \mathrm{H}_{2} \mathrm{O}+6 \mathrm{NADP}^{+}+9 \mathrm{ADP}+8 \mathrm{P}_{\mathrm{i}}$ Glyceraldehyde 3-phosphate

The analysis and calculation is simplified if we use the shortcut introduced in eq.(13)

$$
\begin{aligned}
& 3 \mathrm{CO}_{2}+6 \text { " } \mathrm{H}_{2} \text { " }{ }_{\text {NADPH }}+9 \mathrm{ATP}+8 \mathrm{H}_{2} \mathrm{O} \rightarrow \mathrm{C}_{3} \mathrm{H}_{5} \mathrm{O}_{3} \mathrm{PO}_{3} \mathrm{H}+3 \mathrm{H}_{2} \mathrm{O}+9 \mathrm{ADP}+8 \mathrm{P}_{\mathrm{i}} \\
& 3(-394) \quad 6 \times 61 \quad 8 \times 37+1 \times(-2293-(-1425)) \quad-1077 \quad 3 \times(-156) \\
& \text { Totals: } \quad-1388 \\
& -1545 \Rightarrow \Delta_{\mathrm{r}} G^{\mathrm{o} "}=-157 \mathrm{~kJ} / \mathrm{mol}
\end{aligned}
$$

Without extraneous ATP, this further simplifies to

$$
\begin{aligned}
& 3 \mathrm{CO}_{2}+6 \text { “" } \mathrm{H}_{2} \text { ” }{ }_{\mathrm{NADPH}}+\mathrm{ATP} \rightarrow \mathrm{C}_{3} \mathrm{H}_{5} \mathrm{O}_{3} \mathrm{PO}_{3} \mathrm{H}+3 \mathrm{H}_{2} \mathrm{O}+\mathrm{ADP} \\
& 3(-394)+6 \times 61 \quad-2293 \quad-1077 \quad 3 \times(-156)-1425 \Rightarrow \Delta_{\mathrm{r}} G^{\mathrm{o} "}=+139 \mathrm{~kJ} / \mathrm{mol}
\end{aligned}
$$

Leaving out the phosphate, with glyceraldehyde as the product, we obtain

$$
\begin{array}{lll}
3 \mathrm{CO}_{2}+6 \text { " } \mathrm{H}_{2} \text { " }{ }_{\text {NADPH }} \rightarrow & \left(\mathrm{CH}_{2} \mathrm{O}\right)_{3}+3 \mathrm{H}_{2} \mathrm{O} \\
3(-394) \quad 6 \times 61 & \sim-187 \quad 3 \times(-156) \Rightarrow \Delta_{\mathrm{r}} G^{\mathrm{o} "} \approx+161 \mathrm{~kJ} / \mathrm{mol}
\end{array}
$$

We can also consider the ultimate formation of glucose:

$$
\begin{aligned}
& 6 \mathrm{CO}_{2}+12 \text { " } \mathrm{H}_{2} \text { " } \\
& \Delta_{\mathrm{r}} G^{\mathrm{o}^{\prime}}: \quad 6(-394)=-2364 \quad 12 \times(-17) \quad-911 \quad 6 \times(-237) \Rightarrow \Delta_{\mathrm{r}} G^{\mathrm{o}^{\prime}}=+235 \mathrm{~kJ} / \mathrm{mol} \\
& \Delta_{\mathrm{r}} G^{\mathrm{o}^{\circ}:} \quad 6(-394)=-2364 \quad 12 \times(61) \quad-427 \quad 6 \times(-156) \Rightarrow \Delta_{\mathrm{r}} G^{\mathrm{o}^{\prime \prime}}=+269 \mathrm{~kJ} / \mathrm{mol}
\end{aligned}
$$

The free energy change is quite minor ( $10 \%$ of the free energy of combustion of glucose), and its sign can be attributed to entropy (linking small molecules into a large one reduces their entropy). This disproves the 
notion that a lot of energy must be put in to convert a "highly oxidized molecule" like $\mathrm{CO}_{2}$ to a "more reduced" carbohydrate. When $\mathrm{O}_{2}$ is not involved, the energy changes are small.

The energetics of this net reaction of the Calvin cycle have rarely been analyzed, probably since its negative enthalpy and small free-energy change cannot be rationalized in the dogmatic view that fuel molecules store the chemical energy. Instead, the canonical combustion reaction of glucose, or its reverse, is sometimes brought up in this context, ${ }^{16}$ confusing the energetic picture. A chemical process with similar reactants as in eq. (28), the reverse of eq.(3c) with $\Delta_{\mathrm{r}} G^{\mathrm{o}^{\prime}}=-804 \mathrm{~kJ} / \mathrm{mol}$, is indeed used by methanogens to produce energy, qualitatively confirming that the reaction of $\mathrm{CO}_{2}$ and $\mathrm{H}_{2}$ to form "more reduced molecules" can be energetically downhill.

Review of biochemical energy in textbooks. A reviewer of this paper requested documentation that the misconceptions regarding biochemical energetics identified in this work are pervasive in current textbooks. The following quotes from eight biochemistry or molecular biology textbooks and three specialized books on biochemical thermodynamics provide evidence for this, in particular that $\mathrm{O}_{2}$ had not been identified as the molecule storing most biochemical energy. Particularly relevant phrases are highlighted in bold.

In Lehninger Principles of Biochemistry, ${ }^{12}$ Figure 1-25 shows "energy transformations in living organisms": At the top, "Potential energy" is associated with sunlight and "Nutrients in environment (complex molecules such as sugars, fats)" and in the caption it is explained that "the potential energy of the complex nutrient molecules decreases." $\mathrm{O}_{2}$ is not shown at all in this figure. The text states that "Organisms obtain energy from their surroundings ... they take up chemical fuels (such as glucose) from the environment and extract energy by oxidizing them." On p.22, it is asserted that "organisms ... obtain the energy they need by oxidizing the energy-rich products of photosynthesis stored in plants, then passing the electrons thus acquired to atmospheric $\mathrm{O}_{2} \ldots$... On p. 25, it is stated that cells are "extracting energy (from nutrients such as glucose)...", on p. 357 that "Fats and oils are the principal forms of energy in many organism." and on p. 555 that: "Electron transfer from NADH to $\mathrm{O}_{2}$ in mitochondria provides the energy for synthesis of ATP...".

Alberts et al. ${ }^{17}$ in Molecular Biology of the Cell assert very clearly (p. 54) that "All animal and plant cells are powered by energy stored in the chemical bonds of organic molecules... In both plants and animals, energy is extracted from food molecules ...". The caption of Figure 2-18 refers to conversion of "... sunlight into chemical-bond energy in sugars and other organic molecules." According to p. 73, "The constant supply of energy that cells need ... comes from the chemical-bond energy in food molecules." Their Figure 2-20 shows that reduced molecules like $\mathrm{CH}_{4}$ are high in energy because of more electrons on carbon. This is disproved in the present work. The crucial highenergy molecule, $\mathrm{O}_{2}$, is missing from this picture. On p. 67, the authors assert that "Activated carriers are specialized to carry electrons held at a high energy level (sometimes called "high-energy" electrons). The most important of the electron carriers are $\mathrm{NAD}^{+} \ldots$ and $\ldots \mathrm{NADP}^{+}$" [this is extra wrong, because these forms of the molecules are obviously depleted of electrons]. "Each picks up a "packet of energy" corresponding to two electrons plus a proton." In the same vein, on p. 68 one can read that "NADPH operates ... supplying the high-energy electrons needed to synthesize energy-rich biological molecules." The observation on p. 81 that "animals ... cannot convert fatty acids to sugars." is explained in this work: fatty acids are low-energy molecules. 
Becker's World of the Cell ${ }^{18}$ clearly states that "Chemotrophs .. must depend completely on energy that has been packaged into oxidizable food molecules." close to Figure 5.4, whose caption ends in "Solar energy is used to reduce low-energy inorganic compounds to high-energy organic compounds, which are used by both phototrophs and autotrophs", while the main high-energy compound in the biosphere is inorganic $\mathrm{O}_{2}$.

Berg, Tymoczko, and Stryer in Biochemistry", p. 444, show a diagram "Fuel (carbohydrates, fats) $\rightarrow \mathrm{CO}_{2}+\mathrm{H}_{2} \mathrm{O}+$ useful energy" without $\mathrm{O}_{2}$, even though the oxygen-rich products imply its contribution. Clearly, the dominant contribution of $\mathrm{O}_{2}$ to the useful energy was not recognized here. For fats, the reaction is impossible without $\mathrm{O}_{2}$ and would not produce energy. According to p. 453: "Energy from foodstuffs is extracted in three stages", which attributes chemical energy to organic molecules, not $\mathrm{O}_{2}$. The text on p. 454: "The reduced forms of these carriers then transfer their high-potential electrons to $\mathrm{O}_{2}$." attributes energy to species other than $\mathrm{O}_{2}$, specifically reduced species. Table 15.2 asserts that NADH, $\mathrm{NADPH}, \mathrm{FADH}_{2}$, and $\mathrm{FMNH}_{2}$ carry electrons, while they obviously carry and transfer $\mathrm{H}$ atoms. On p. 546, the energetics of aerobic respiration are summarized: "In oxidative phosphorylation, the electrontransfer potential of NADH or $\mathrm{FADH}_{2}$ is converted into the phosphoryl-transfer potential of ATP." without mentioning $\mathrm{O}_{2}$.

Campbell Biology ${ }^{20}$ on p. 142 refers to "extracting the energy stored in sugars and other fuels". Further, (p.143) “... glucose and other organic fuels are broken down in the presence of oxygen ... Energy that was stored in the organic molecules becomes available ..." "these complex molecules, such as glucose, are in high in chemical energy."

Karp's Cell and Molecular Biology ${ }^{11}$ states very clearly on p. 110 that "Carbohydrates are rich in chemical energy ... Fats contain even greater energy per unit weight because the contain strings of more reduced $\mathrm{CH}_{2}$ units.", while it has been shown in this work that reduced molecules like fats are low in energy.

Harper's Illustrated Biochemistry ${ }^{21}$ on p. 122 states: "Aerobic organisms are able to capture a far greater proportion of the available free energy of respiratory substrates..." attributing chemical energy not to $\mathrm{O}_{2}$ but to other reactants. Table 18-1 on p. 177 incorrectly attributes the energy of $\mathrm{O}_{2}$ to the citricacid cycle.

Voet \& Voet in Biochemistry ${ }^{22}$ initially do not seem to attribute biochemical energy to specific molecules. However, on p. 574 they write " $N A D H$ thereby functions as an energy rich electron-transfer coenzyme." This implicitly attributes the energy of the overall reaction in the respiratory chain, between $\mathrm{NADH}$ and $\mathrm{O}_{2}$, to $\mathrm{NADH}$ rather than $\mathrm{O}_{2}$. In Figure 22.9, the electron-transport chain is shown in terms of standard reduction potentials and associated "energy levels", with $\mathrm{O}_{2}$ at the bottom.

Physical chemists routinely set the energy of $\mathrm{O}_{2}$ to zero (since it is the element in its standard state). This makes the bond energy of $\mathrm{O}_{2}$ invisible. They rely on long tables of meaningless numbers (enthalpies of formation etc.). Therefore they have been unable to explain the energetics of combustion. Both fire scientists and some biochemists have empirically discovered the proportionality of the heat of combustion with $\mathrm{O}_{2}$ consumed ("oxygen combustion calorimetry"). But they attributed it to "the number of available electrons per mole of substrate" ${ }^{23}$ or argued that "the energetic processes are the result of breaking either carbon-carbon or carbon-hydrogen bonds, and these have similar bond strengths" ${ }^{24}$. These arguments are all about the fuel, not $\mathrm{O}_{2}$. 
It is the same in the specialized literature. Bioenergetics - Its Thermodynamic Foundations by Garby \& Larson ${ }^{9}$ has only one entry on oxygen in their index. Section 4.5.2 on combustion does not explain the energetics, but is just based on the tabulated heat of formation of glucose. The authors do not explain why carbohydrates have only $40 \%$ of the heat of combustion of fat, which the oxygen-based combustion energetics can explain quantitatively (because carbohydrates contain oxygen, they use less $\mathrm{O}_{2}$ in their combustion). Thermodynamics of Biochemical Reactions by R. A. Alberty ${ }^{14}$ has only two minor index entries on oxygen and none on combustion, respiration, or (an)aerobic. In his tables, $\mathrm{O}_{2}(\mathrm{~g})$ is always at $0.00 \mathrm{~kJ} / \mathrm{mol}$.

Energy Flow in Biology by H. J. Morowitz ${ }^{25}$ has on its cover (and on p. 80) a diagram of energy flow from the sun to various organisms without $\mathrm{O}_{2}$ (while $\mathrm{CO}_{2}$ is shown). On p. 61, the author writes about (organic) "molecules, which are energy-rich". This is followed by a long discussion of bond energies in organsims, including the energy of covalent bonds in E. coli. The "production of oxygen" in photosynthesis is mentioned but there is no discussion of the energetics of $\mathrm{O}_{2}$. (One has to count electronpair bonds, whose number does not change in combustion, for a generalized bond-energy analysis and in order to see the special status of $\mathrm{O}_{2}$.) "the enthalpy change is very large for this case, since the reactants are in a highly oxidized state which, in general, corresponds to [a] large free energy of formation." That is the conventional picture without $\mathrm{O}_{2}$. On p. 108, Morowitz discusses heats of combustion without any mention of the special role of $\mathrm{O}_{2}$. He also does not explain why carbohydrates have only $40 \%$ of the heat of combustion of fat.

Correcting textbooks: an example. Can the needed dramatic revisions of biochemical energetics be incorporated into existing textbooks? The following example, a more correct rephrasing of an introductory text on chemical energy and electron-transfer chains, from Lehninger, ${ }^{12}$ p. 528 , shows that this is manageable. Significantly revised parts are underlined.

"Every time we use a motor, a refrigerator, or a battery charger, we use the flow of electrons to perform work. In the circuit that powers a motor, the source of electrons can be a battery in which a chemical reaction can only occur if electrons flow, usually through a metallic wire, from the half reaction at one pole of the battery, through the motor, to the half reaction at the other pole of the battery. The chemical force driving the electrons is manifested as the electromotive force, emf. The emf (typically a few volts) can accomplish work if an appropriate energy transducer - in this case a motor - is placed in the circuit. The motor can be coupled to a variety of mechanical devices to do useful work.

Living cells have analogous biological "circuits", with chemical reactions that convert high-energy reactants to lower-energy products driving the flow of electrons or protons. The energy released in exergonic reactions is channeled to drive the flow of electrons, avoiding loss as heat, in an electrontransfer chain involving a series of electron-carrier intermediates. The electrons provide energy to a variety of molecular energy transducers (enzymes and other proteins) that do biological work. In the mitochondrion, for example, membrane-bound enzymes couple electron flow, driven by energy that had been stored in the weak double bond of $\mathrm{O}_{2}$, to the production of a transmembrane proton concentration gradient and electrical potential, performing osmotic and electrical work. The resulting proton gradient has potential energy, sometimes called the proton-motive force by analogy with electromotive force. Another enzyme, ATP synthase in the inner mitochondrial membrane, uses the proton motive force to do mechanical work and convert it to chemical energy in ATP synthesized from ADP and $\mathrm{P}_{\mathrm{i}}$ as protons flow 
spontaneously across the membrane. Similarly, membrane-localized enzymes in E. Coli use chemical energy to drive electron and proton flow, which is then used to power flagellar motion. The principles of electrochemistry that relate free-energy changes in a battery to electrical energy driving a motor or charging a second battery apply with equal validity to the molecular processes accompanying electron and proton flow in living cells."

\section{References Cited}

(1) Schmidt-Rohr, K. Why Combustions Are Always Exothermic, Yielding About $418 \mathrm{~kJ}$ per Mole of $\mathrm{O}_{2}$. J. Chem. Educ. 2015, 92, 2094-2099.

(2) McMurry, J.; Fay, R. C. Chemistry, $3^{\text {rd }}$ ed.; Pearson: Upper Saddle River, NJ, 2004.

(3) Darwent, B. d. Bond Dissociation Energies in Simple Molecules. Nat. Stand. Ref. Data. Ser., Nat. Bur. Stand. (U.S.) 1970, 31, 1-52.

(4) Borden, W. T.; Hoffmann, R.; Stuyver, T.; Chen, B. Dioxygen: What Makes This Triplet Radical Kinetically Persistent? J. Am. Chem. Soc. 2017, 139, 9010-9018.

(5) Murphy, L. R.; Meek, T. L.; Allred, A. L.; Allen, L. C. Evaluation and Test of Pauling's Electronegativity Scale. J. Phys. Chem. A 2000, 104, 5867-5871.

(6) Pauling, L. The Nature of the Chemical Bond; Cornell University Press: Ithaca, 1939.

(7) Schmidt-Rohr, K. How Batteries Store and Release Energy: Explaining Basic Electrochemistry. J. Chem. Educ. 2018, 95, 1801-1810.

(8) Oxtoby, D. W.; Gillis, H. P.; Butler, L. J. Principles of Modern Chemistry, $8^{\text {th }}$ ed.; CENGAGE Learning: Boston, MA, 2015.

(9) Garby, L.; Larsen, P. Bioenergetics; Cambridge University Press: Cambridge, 2003.

(10) Domalski, E. S. Selected Values of Heats of Combustion and Heats of Formation of Organic Compounds Containing the Elements C, H, N, O, and P. J. Phys. Chem. Ref. Data 1972, 1, 221-277.

(11) Karp, G. Cell and Molecular Biology, $7^{\text {th }}$ ed.; Wiley: Hoboken, NJ, 2013.

(12) Nelson, D. L.; Cox, M. M. Lehninger Principles of Biochemistry, $6^{\text {th }}$ ed.; Worth Publishers: New York, 2013.

(13) Alberty, R. A.; Silbey, R. J. Physical Chemistry, $2^{\text {nd }}$ ed.; Wiley: New York, 1997. p.Table 8.3.

(14) Alberty, R. A. Thermodynamics of Biochemical Reactions; Wiley: Hoboken, NJ, 2003. p.211-213.

(15) Bockris, J. O.; Reddy, A. K. N.; Gamboa-Aldeco, M. Modern Electrochemistry 2A, Fundamentals of Electrodics, $2^{\text {nd }}$ ed.; Kluwer Academic / Plenum: New York, 2001.

(16) Taiz, L.; Zeiger, E.; Møller, I. M.; Murphy, A. Plant Physiology and Development, $6^{\text {th }}$ ed.; Oxford University Press: Oxford, 2014. Topic 8.4: Energy Demands for Photosynthesis in Land Plants.

(17) Alberts, B.; Johnson, A.; Lewis, J.; Morgan, D.; Raff, M.; Roberts, K.; Walter, P. Molecular Biology of the Cell, $6^{\text {th }}$ ed.; Garland Science / Taylor \& Francis: New York, 2008.

(18) Hardin, J.; Betoni, G. P. Becker's World of the Cell, 9 $9^{\text {th }}$ ed.; Pearson: Boston, 2015.

(19) Berg, J. M.; Tymoczko, J. L.; Stryer, L. Biochemistry, 7th ed.; W. H. Freeman: New York, 2012.

(20) Reece, J. B.; Urry, L. A.; Cain, M. L.; Wasserman, S. A.; Minorsky, P. V.; Jackson, R. B. Campbell Biology, $7^{\text {th }}$ ed.; Pearson / Benjamin Cummings: Boston, 2011.

(21) Bender, D. A.; Botham, K. M.; Kennelly, P. J.; Rodwell, V. W.; Weil, P. A. Harper's Illustrated Biochemistry, 29 ${ }^{\text {th }}$ ed.; McGraw Hill: New York, 2012.

(22) Voet, D.; Voet, J. G. Biochemistry, $3^{\text {rd }}$ ed.; Wiley: Hoboken, New Jersey, 2004.

(23) Payne, W. J. Energy Yields and Growth of Heterotrophs. Annu. Rev. Microbiol. 1970, 24, 17-52.

(24) Huggett, C. Estimation of Rate of Heat Release by Means of Oxygen Consumption Measurements. Fire Mater. 1980, 4, 61-65.

(25) Morowitz, H. J. Energy Flow in Biology; Ox Bow Press: Woodbridge, CT, 1979. 